\title{
CONTRIBUCIÓN DELAS REVISTAS ACADÉMICAS Y LOS PREMIOS NOBEL DEECONOMÍA AL MEJORAMIENTO DEL CAMPO FINANCIERO EN COLOMBIA
}

\section{Julián Monroy D'Croz}

\section{RESUMEN:}

El presente artículo muestra, en primer lugar, las principales ventajas de la base de datos en línea http:// www.jstor.org como fuente de consulta e investigación para programas académicos en el campo financiero. Además, plantea la influencia de las publicaciones y de las universidades americanas en el diseño de la educación doctoral en el área financiera, apoyándose principalmente en un estudio realizado por Corrado y Ferris (1997), entre otras investigaciones similares. Este análisis se estableció como marco de referencia para mejorar la calidad de los programas académicos en el ámbito financiero y, más específicamente, en el área de Mercados Financieros para el caso colombiano. En segundo lugar, presenta un breve análisis sobre la relevancia de los premios Nobel de Economía en el área financiera, junto con una breve síntesis de la evolución del pensamiento económico y de la Economía Financiera. Finalmente, concluye que existe una fuerte relación entre las publicaciones financieras líderes, las universidades más importantes y la contribución de los premios en Ciencias Económicas a este campo.

\section{ABSTRACT}

The following article shows, in first place, the main advantages of the on-line data base http://www. jstor.org as an important consultation and research source for academic programs in the financial domain. Besides that shows journal and american universities influence on the design of finance doctoral education, supporting that mainly in a survey developed by Corrado y Ferris (1997), between various similar researches; and establishing this analysis as a model of reference in academic syllabus quality improvement in the financial domain, and more specifically, in Financial Markets area for the colombian case. It also presents a brief analysis about the relevance of Economics Nobel awards in the financial domain together with a short synthesis of the evolution of economic thought and Financial Economics. Finally, it concludes that there exists a strong relationship between finance leader journals, the most important universities and the contribution of Economic Science's prizes in this domain.

\section{PALABRAS CLAVE:}

Publicaciones académicas, programa académico en el área financiera, Economía Financiera, Premio Nobel en Ciencias Económicas.

\section{KEYWORDS:}

Academic publications, Programs Academic in the Financial Area, Financial Economy, Nobel Prize in Economic Sciences. 


\section{INTRODUCCIÓN}

En el presente artículo se muestran los beneficios y ventajas al utilizar una base de datos electrónica centralizada para consultar revistas académicas (artículos de publicación científico-académica). También se quiere mostrar cómo pueden contribuir dichas publicaciones científicas a mejorar la calidad de los programas en el campo financiero y, más específicamente, en la categoría de estudio de los mercados financieros ${ }^{1}$; se analizan dos aspectos muy importantes: primero, la influencia que tienen dichas revistas en el diseño de la educación doctoral en finanzas; y segundo, el aporte de los premios Nobel de Economía, a través de estas publicaciones, al campo financiero. En el primer caso se analiza principalmente un estudio desarrollado por Corrado y Ferris (1997), que muestra la influencia de las revistas en el diseño de programas de doctorado en Finanzas para 33 universidades americanas. Lo anterior sirve como modelo de referencia en el desarrollo de cursos de pregrado y postgrado en el área de mercados financieros para el caso de algunas universidades colombianas, por la sencilla razón de que los programas doctorales, al menos en muchas de las universidades americanas, están o tratan de estar en la frontera del conocimiento. En el segundo caso se presenta la contribución directa de los premios Nobel de Economía al campo financiero, específicamente al área

\section{RESEÑA DEAUTOR}

Julián Monroy D'croz. Profesor de finanzas, Facultad de Ciencias de la Administración, Universidad del Valle. de Mercados Financieros. En el presente artículo se investigan cuatro problemas en la docencia e investigación universitaria en el campo financiero de la actualidad: ¿cuáles son las universidades más importantes en el campo financiero en el mundo?, ¿cuáles son las revistas académicas más importantes en el campo financiero?, ¿cuáles son los premios Nobel de Economía que han hecho aportes directos al campo financiero? y ¿cuáles son los desarrollos teóricos más relevantes de la Economía Financiera?

Con lo anterior, también se muestra un listado bibliográfico general de las diferentes publicaciones que han marcado fuertes líneas de investigación a través de los años y de las cuales algunas han sido consideradas como investigaciones de mención honorífica por la comunidad académica internacional y han marcado, en su época, un cambio de paradigma importante en el campo financiero.

El presente artículo está organizado de la siguiente manera: en la primera parte se desarrolla la importancia académica del uso de una base de datos electrónica; en la segunda parte se discute la influencia de las revistas y de las universidades estadounidenses en la educación doctoral en finanzas; en la tercera parte se expone la importancia de los premios Nobel de Economía y sus aportes al campo financiero, así como la síntesis del pensamiento económico y la Economía Financiera; en la cuarta parte se presenta una propuesta curricular en el área de Mercados Financieros para Colombia; finalmente se presenta las conclusiones generales de la presente investigación.

\footnotetext{
1. A la categoría de mercados financieros pertenecen seis principales áreas de investigación: valuación de activos, selección de portafolio, información y eficiencia de mercado, representación y valuación de futuros, mercados financieros internacionales y regulación y política de gobierno (clasificación realizada por Borokhovich y otros. (1994)).
} 


\section{IMPORTANCIA ACADÉMICA DE LA BASE DE DATOS ELECTRÓNICA \\ http://www.jstor.org}

Journal storage o jstor ${ }^{2}$ es una base de datos electrónica especializada en revistas académicas. Esta base de datos se conforma básicamente a partir de seis colecciones bibliográficas de disciplinas específicas; estas son: Ecología y Botánica (29 títulos), Ciencia General (7 títulos), Lenguaje y Literatura (52 títulos), Matemáticas y Estadística (31 títulos), Música (32 títulos) y Negocios (49 títulos) ${ }^{3}$; además existen otras 28 colecciones, cada una con sus respectivos títulos, las cuales tienen revistas propias y compartidas de las seis colecciones de disciplinas específicas. La colección de Negocios se especializa en revistas de economía, administración y finanzas principalmente. El acceso a dicha base se hace en línea desde cualquier computadora que esté conectada a una red universitaria que tenga suscripción a dicha base. En la colección de negocios, incluidos los subgrupos pertenecientes a un tipo de publicación específica, existen actualmente 65 revistas 4 académicas de publicación científica que abarcan las áreas de riesgo, seguros, mercadotecnia, administración, contaduría, economía, industrial, negocios, finanzas, organizaciones, investigación de operaciones, estadística y econometría. Las fechas de dichas publicaciones van, en términos generales, desde los años de iniciación de dichas publicaciones, hasta 1998 a 2002 o hasta la fecha de finalización de algunas publicaciones ${ }^{5}$. La gran mayoría de las revistas que contiene dicha base de datos está publicada en inglés (también se encuentran algunas pocas publicaciones en francés y otros idiomas) y se pueden descargar en formato pdf.

2. Jstor (http://www.jstor.org) es una organización independiente sin ánimo de lucro, encargada de creary preservar un archivo digital de las publicaciones científicas académicas. Se tiene en cuenta esta base de datos en la presente investigación, en lugar de otras, por ser una de las más ampliamente utilizadas y con mayor cantidad de publicaciones en el campo financiero.

3. La lista completa de todas las revistas que hacen parte de http://www.jstor.org asciende, en la actualidad, a más o menos 440 cuadernos de publicación científica.

4. Nota: el período de años corresponde a las fechas en que se puede conseguir dichas publicaciones a través de jstor (los datos están actualizados hasta diciembre de 2004; cada nuevo año se suma un año adicional a la base). Academy of Management Journal 1963-1998, Journal of the Academy of Management 1958-1962, Academy of Management Review 1976-1998, Accounting Review 1926-1998, Administrative Science Quarterly 1956-2000, American Economic Review 1911-2000, American Economic Association Quarterly 1908-1910, Publications of the American Economic Association 1886-1907, Brookings Papers on Economic Activity 1970-2000, Brookings Papers on Economic Activity, Microeconomics 1989-1998, Canadian Journal of Economics 1968-2000, Canadian Journal of Economics and Political Science 1935-1967, Contributions to Canadian Economics 19281934, Econometrica 1933-2000, Economic History Review 1927-1998, Economic Journal 1891-1998, Economica 1921-1998, Industrial and Labor Relations Review 1947-1998, International Economic Review 1960-1998, Journal of Accounting Research 1963-2000, Journal of Applied Econometrics 1986-1998, Journal of Business 1954-2001, Journal of Business of the University of Chicago 1928-1953, University Journal of Business 19221927, Journal of Consumer Research 1974-2000, Journal of Economic History 1941-1998, Journal of Economic Literature 1969-2000, Journal of Economic Abstracts 1963-1968, Journal of Economic Perspectives 1987-2000, Journal of Finance 1946-2000, Journal of Financial and Quantitative Analysis 1966-1999, Journal of Human Resources 1966-2001, Journal of Industrial Economics 1952-1998, Journal of International Business Studies 1970-2000, Journal of Labor Economics 1983-2001, Journal of Money, Credit and Banking 1969-1998, Journal of Organizational Behavior 1988-1998, Journal of Occupational Behavior 1980-1987, Journal of Political Economy

Journal storage o jstor es una base de datos electrónica especializada en revistas académicas. 
A partir de enero de 1997 se han vinculado a los servicios de jstor, solamente en los Estados Unidos, 1.252 instituciones académicas estadounidenses; y a partir de 2000 se ha ido incrementando las vinculaciones por parte de instituciones académicas extranjeras (véase http://www.jstor. org/info).

La colección de Negocios es de vital importancia para las facultades universitarias que albergan los programas de pregrado y postgrado de Economía, Administración de Empresas, Contaduría Pública, Finanzas, Ingeniería Industrial y Organizaciones entre otros, puesto que contiene muchas investigaciones científicas importantes en la mayoría de las áreas de dichos programas. Aunque en Colombia no existe una educación doctoral formal en finanzas ${ }^{6}$ y son muy pocos los programas de Maestría en Finanzas. Por esto, el presente documento quiere aportar algunas ideas para contribuir al mejoramiento de la calidad de los cursos, tanto en pregrado como en postgrado, en el campo financiero.

De acuerdo con Guthrie (2001), se espera que en el futuro cercano las instituciones académicas tengan preferencia por pagar una base de datos digital para archivar todas las publicaciones científicas pertinentes, más que por preservar físicamente una biblioteca. Varias razones justifican lo anterior: en primer lugar, todos los documentos residen en un servidor al que se puede acceder por cualquier usuario autorizado desde cualquier lugar del mundo; esto permite que la biblioteca local de cada universidad no tenga la necesidad de tener una costosa infraestructura de grandes servidores dentro del campus universitario dedicada exclusivamente a guardar, mantener y enviar toda esta documentación. En segundo lugar, se espera que cada vez aumente más rápido y en mayor canti-

1892-2000, Journal of Risk and Insurance 1964-2001, Journal of Insurance 1957-1963, Journal of the American Association of University Teachers of Insurance 1937-1956, Proceedings of the Annual Meeting (American Association of University Teachers of Insurance) 1933-1935, Journal of the Operational Research Society 19781998, Operational Research Quarterly 1970-1977, OR 1953-1969, Operational Research Quarterly 1950-1952, Management Science 1954-2000, Management Technology 1960-1964, Managerial and Decision Economics 1980-1998, Marketing Science 1982-2000, MIS Quarterly 1977-1998, Operations Research 1956-2000, Journal of the Operations Research Society of America 1952-1955, Organization Science 1990-2000, Oxford Economic Papers 1938-1997, Quarterly Journal of Economics 1886-1998, RAND Journal of Economics 1984-1998, Bell Journal of Economics 1975-1983, Bell Journal of Economics and Management Science 1970-1974, Review of Economic Studies 1933-2000, Review of Economics and Statistics 1919-1998, Review of Financial Studies 19882000, Strategic Management Journal 1980-1998.

5. En el caso de la Universidad del Valle, ésta está inscrita a dicha base de a partir de enero de 2004.

6. Consulta llevada a cabo a través de la revisión de los programas de postgrado de las universidades más importantes de Colombia, entre las que figuran: Universidad de los Andes, Universidad Javeriana, Universidad EAFIT, Universidad Externado, Universidad Nacional, Universidad de Antioquia, Universidad del Valle, Universidad San Buenaventura, Universidad ICESI, Universidad del Cauca, Universidad Libre, entre varias. Para mayor información se puede consultar: Guía profesional postgrados 2007, Bogotá: Publi Legis, y los sitios web de dichas instituciones.

En el presente artículo no se pretende hacer ninguna mención o estudio en particular, de ninguna facultad donde se dicten programas en el campo financiero, más bien pretende ser un documento que sirva como una ayuda para mejorar la calidad de dichos programas en las facultades interesadas. 
dad la producción intelectual científica, la cual es difundida principalmente a través de las revistas académicas, lo que, a mediano y largo plazo, sería muy costoso de mantener y archivar físicamente, puesto que implicaría gastos como pagar más empleados para que se encarguen de archivar los documentos, construir más espacios en las bibliotecas, etcetera. En tercer lugar, las organizaciones editoras de las revistas han estado incrementando cada vez más el envío de contenidos electrónicos (revistas electrónicas), especialmente en los países fuera de Estados Unidos, debido a que la suscripción a la revista electrónica (contenido digital) y los documentos llegan de inmediato, es más económica con relación a la suscripción a la revista física (contenido de papel).

A finales de la década de 1990, el costo anual de acceso a 7.700 volúmenes de revistas (únicamente la colección de Ciencias y Artes), era de US \$6.500 para el sistema tradicional contra US $\$ 2.000$ (en el caso de las instituciones universitarias más pequeñas) para el acceso al contenido electrónico a través de jstor. Por otra parte los costos de mantenimiento y archivado de cada volumen se han estimado en US \$16 para el sistema tradicional y US \$4 para el sistema remoto Guthrie: (2001, 60). En la actualidad, estas relaciones han mejorado, en términos económicos, significativamente.

Otro aspecto bastante importante consiste en que al bajar los costos, el acceso por parte de académicos y estudiantes a estos materiales ha venido aumentando especialmente en la consulta de materiales antiguos, lo cual sugiere la realización de adecuados y suficientes desarrollos teóricos en los diferentes campos de investigación; se estima que en la actualidad éstos estén imprimiendo más de siete millones de artículos de la base de datos anualmente alrededor del mundo.

Jstor tiene la gran ventaja de reducir los costos de mantenimiento del material y además ofrecer un acceso rápido, seguro y masivo ${ }^{7}$ a toda la información, aspecto que sería imposible a través del sistema tradicional de archivo de biblioteca. De esta manera jstor funciona más como una base de datos que como un archivo, la cual administra centralmente toda la información pertinente con la intención de preservarla y permitir acceso a todos sus contenidos a muy bajos $\operatorname{costos}^{8}$.

Una desventaja que presenta jstor es la "pared móvil" (moving wall). Este es un aspecto que está estipulado en el acuerdo de licencia de jstor con los editores de las diferentes revistas y consiste en que, dependiendo de la revista, existe un lapso de tiempo entre los dos a los cinco años más recientes, en que no es posible tener acceso a las publicaciones a través de jstor. Por ejemplo, la "pared móvil" del Journal of Finance es de tres años, es decir, que en el presente a través de la base de datos jstor, solamente se puede tener acceso hasta las publicaciones de 2003. Para todas las publicaciones, cada vez que pase un año calendario se adiciona

7. Varios individuos pueden estar descargando el mismo artículo, en el mismo momento y en el mismo lugar o en diferentes lugares del mundo.

8. También existen otras bases de datos electrónicas muy completas, de carácter comercial y académico, para consultar revistas y textos académicos como http://www.sciencedirect.com, http://www.elsevier.com, http://www.ebsco.org. 
un nuevo año, el cual estará disponible en la base de datos de jstor ${ }^{9}$.

A pesar del gran tamaño de la base de datos jstor (440 revistas), hacen falta varias revistas importantes pertenecientes al campo financiero, las cuales componen un importante recurso bibliográfico para la docencia e investigación universita-
Dicha investigación evalúa 101 diferentes programas de Doctorado en Finanzas, reportados por

33 escuelas de negocios acreditadas de Estados Unidos. ria $^{10}$. Entre estas revistas se encuentran las siguientes: Financial Analysts Journal, Journal of Financial Economics, Journal of Empirical Finance, Journal of Derivatives, Journal of Forecasting, Journal of Banking and Finance, Journal of Econometrics, Journal of Monetary Economics, Journal of Business Ethic, Journal of Future Markets, Quantitative Finance, Journal of Financial Markets, Mathematical Finance, Macroeconomics Annual, Journal of Business and Economic Statistics, Journal of Financial Econometrics, Journal of Portfolio Management, Journal of Risk and Uncertainty, Journal of Psychology and Financial Markets y European Economic Review.

\section{INFLUENCIA DE LAS REVISTAS Y DE LAS UNIVERSIDADES ESTADOUNIDENSES EN LA EDUCACIÓN DOCTORAL EN FINANZAS}

Corrado y Ferris (1997) desarrollaron una investigación bastante interesante que muestra la influencia de las publicaciones científicas académicas en el diseño de una educación doctoral en finanzas.
Dicha investigación evalúa 101 diferentes programas de Doctorado en Finanzas, reportados por 33 escuelas de negocios acreditadas de Estados Unidos ${ }^{11}$. De este grupo se compilan 1.031 artículos de publicación científica citados por los programas. Dichos artículos se encuentran contenidos en 17 revistas de finanzas, economía y contaduría, principalmente, de los cuales cinco revistas tienen el $82 \%$ de la totalidad citada.

Las escuelas más representativas de dicha lista que tienen la mayor cantidad de artículos publicados - para el período 1989 - 1993-son:

1. University of California, Los Angeles (48 artículos).

2. Ohio State University (46 artículos).

3. Columbia University (41 artículos).

4. Rochester University (33 artículos).

5. British Columbia University (29 artículos).

Las anteriores universidades están supervisadas por la Association to Advance Collegiate School of Business (AACSB). Esta es la organización más importante mundialmente, dedicada a la acreditación, promoción y mejoramiento de la educación y formación en el campo de los negocios, finanzas y gestión empresarial. Esta organización fue creada en 1916 por varias universidades americanas de alto nivel, entre las que se encuentran universidades esta-

9. Este inconveniente, es decir, la falta de documentos con información de frontera (por la carencia de las publicaciones de los años más recientes), puede ser solucionado por las facultades académicas respectivas, al inscribirse electrónicamente-lo cual abarata mucho los costos-a los 3 ó 4 revistas más importantes en cada una de las áreas académicas correspondientes, de esa manera se tendría una gran parte de la información de frontera correspondiente.

10. Aunque estas revistas no se encuentran en jstor, en otras bases de datos interdisciplinarias en línea como Ebsco, Elsevier y Sciencedirect, se pueden encontrar muchas de estas publicaciones.

11. En dicha lista se encuentra London Business School, como única escuela europea con doctorado. 
tales y privadas como Chicago University, University of Nebraska Lincoln, Harvard University, entre otras, y está encargada de acreditar los programas de licenciatura, maestría y doctorado, especialmente en el área de administración de empresas, finanzas y contabilidad.

A finales de 2005 más de $650^{12}$ instituciones educativas estadounidenses, 266 instituciones educativas no estadounidenses ( $\mathrm{de}$ todos los continentes) y 41 organizaciones corporativas sin ánimo de lucro son miembros de la AACSB. Para abril de 2004 existía un total de 480 instituciones académicas acreditadas, siendo 418 de Estados Unidos, y 62 de los demás países ${ }^{13}$. Entre las escuelas acreditadas únicamente para programas de postgrado se encuentran 42 instituciones alrededor del mundo; entre estas están: Chicago, California L.A., Columbia, Harvard, Stanford, Yale, Purdue, Northwestern, London Business School, Duke, HEC-París, Keio, IPADE e Instituto de Empresa.

Existe otro estudio similar desarrollado por Klemkosky y Tuttle (1977) que establece un listado de la contribución en diez años (1966-1976) de los graduados de postgrado de diferentes instituciones universitarias estadounidenses. De acuerdo con la revisión de 11.313 páginas publicadas en el Journal of Finance (JOF), Journal of Financial and Quantitative Análisis (JFQA) y el Journal of Financial Economics (JFE), las cuales corresponden a las revistas más importantes en el área financiera, se estableció que Chicago University tenía un $11,56 \%$ del total de las páginas, seguido por University of California,
Los Angeles (UCLA) (6,06\%), University of California, Berkeley (5,16\%), Harvard University (4,96\%), Stanford University $(4,93 \%)$, Cornell University (3,88\%), Massachusetts Institute of Technology (MIT) (3,82\%). Este estudio mostró la alta producción de los graduados de postgrado, principalmente doctorados, de un pequeño número de universidades estadounidenses; más del 50\% de la totalidad de las páginas fueron contribución únicamente de diez universidades estadounidenses (Klemkosky y Tuttle: 1977, 496). El estudio reveló que el 86\% de los autores habían recibido un título de doctorado y éstos abarcaban el 96\% de la totalidad de las páginas (Klemkosky y Tuttle: 1977, 494). Sin embargo, de acuerdo con Bertin y Zivney (1992) solamente el 5\% de los graduados de doctorado han publicado al menos un artículo por año en alguna revista perteneciente al campo financiero durante un período prolongado de tiempo.

En otro estudio desarrollado nueve años después por Cooley y otros (1986), se observa que el listado de universidades que aportaron investigación a través de sus graduados de programas de doctorado a las tres revistas líderes en el área financiera, permanece muy similar al estudio desarrollado por Klemkosky y Tuttle (1977). Este estudio muestra la contribución de artículos desde el año 1946 hasta 1985 por instituciones académicas, especialmente para el JOF ${ }^{14}$. En la Tabla 1 se puede observar que nuevamente Chicago tiene un alto predominio sobre las demás universidades estadounidenses, en cuanto a investigación y publicaciones se
En otro estudio desarrollado nueve años después por Cooley y otros (1986), se observaque el listado de universidades que aportaron investigación a través de sus graduados de programas de doctorado a las tres revistas líderes en el área financiera, permanece muy similar al estudio desarrollado por Klemkosky y Tuttle (1977).

12. Véase más información al respecto en http://www.isinet.com.

13. Existen solamente cuatro instituciones académicas acreditadas por la AACSB en Sudamérica y, hasta ahora, ninguna en Colombia.

14. Se debe tener en cuenta que el JOF inicia su publicación en 1946, el JFQA en 1966 y JFE en 1973. 
refiere. La importancia de esta lista radica también en que, como se expondrá más adelante, la mayoría de las universidades de esta lista ha obtenido la gran mayoría de los 57 premios Nobel de Economía otorgados hasta 2005. Además muchos de los autores merecedores de un premio Nobel de Economía, especialmente aquellos que han desarrollado contribuciones al campo financiero, han tenido la oportunidad de participar en muchas de estas universidades a lo largo de su carrera profesional como estudiantes, docentes e investigadores.

Tabla 1. Lista de universidades estadounidenses con mayor cantidad de publicaciones. 1946-1985.

\begin{tabular}{|l|c|}
\hline \multicolumn{1}{|c|}{ Unive rsidad } & Cantidad de artículos \\
\hline Chicago University & 200,03 \\
\hline Harvard University & 129,39 \\
\hline MIT & 111,71 \\
\hline Columbia University & 103,78 \\
\hline University of C. Berkeley & 88,04 \\
\hline Stanford University & 74,33 \\
\hline University of Wisconsin & 74,33 \\
\hline University of Michigan & 63,98 \\
\hline Northwestern University & 63,33 \\
\hline New York University & 56,23 \\
\hline University of Pennsylvania & 54,58 \\
\hline Princeton University & 49,92 \\
\hline University of Minnesota & 44,67 \\
\hline
\end{tabular}

Fuente: Cooley y otros (1986).

Nota: se asume, por falta de información actualizada, que este orden continúa muy similar en la actualidad.

El mencionado estudio muestra también un aspecto relevante que consiste en que, para el período de estudio, la Federal Reserve de los Estados Unidos, ocupó el primer lugar en ser el empleador de autores de artículos publicados en el JOF, con $(108,33)$ artículos, seguido de University of Pennsylvania $(139,94)$ y New York University $(86,13)$; lo anterior establece una tendencia a la mezcla entre organizaciones académicas y gubernamentales que con- tribuyen a la investigación académica-científica. Los seis autores que más apariciones han tenido en el JOF entre 1946 y 1985 son, entre una lista de 47 autores, los siguientes: Robert Litzenberger (18), Edwin Elton (16), Martín Gruber (15), Haim Levy (14), Stephen Ross (13) y Richard Roll (12). Estos dos últimos autores, como se expondrá más adelante, hacen parte del grupo más consultado en los programas de doctorado en Finanzas y son autores claves en el desarrollo de la Teoría Moderna del Portafolio.

El estudio desarrollado por Corrado y Ferris (1997) muestran que, aunque las universidades estadounidenses más prestigiosas en el campo financiero como Harvard, MIT, Yale, Princeton, Chicago, Minnesota, Darthmouth, Berkeley, New York, Wisconsin, Stanford, Cornell, Northwestern, Carniege-Mellon, Columbia, Georgetown y Rochester, así como algunas de las universidades más importantes internacionalmente como European School of Management, Asian Institute of Management, Cambridge, HEC-París, IPADE, Oxford, London Business School, Instituto de Empresa, y Manchester, no presentaron sus programas de doctorado en finanzas (la lista de los remitidos a dicho estudio sólo fue de 33 escuelas de negocios acreditadas de 102 peticiones enviadas), la mayoría de los artículos reseñados en el presente documento han sido publicados y utilizados por éstas.

De acuerdo con la investigación desarrollada por Corrado y Ferris (1997), los temas más importantes para los seminarios de doctorado en Finanzas son:

1. Inversiones (38\%).

2. Teoría Financiera (25\%).

3. Finanzas Corporativas (19\%). 
4. Mercados e Instituciones (10\%).

5. Finanzas Internacionales (10\%).

Aunque los anteriores temas son los más comunes, dicha investigación detectó una gran diversidad de temáticas en la selección de los contenidos de los seminarios. Sin embargo, en un estudio más detallado, Borokhovich y otro (1994), se muestra que los temas del campo financiero se dividen en cinco categorías, cada una con sus respectivas áreas de estudio. De acuerdo con esto, la docencia y la investigación en el campo financiero cubren las áreas de finanzas generales, mercados financieros, instituciones financieras, finanzas corporativas y públicas y dinero e interés ${ }^{15}$. La categoría más importante para el énfasis del presente artículo es la de mercados financieros, la cual consta de las siguientes áreas específicas de estudio: valuación de activos, selección de portafolio, información y eficiencia de mercado, representación y valuación de futuros mercados financieros internacionales y regulación y política de gobierno ${ }^{16}$.

Borokhovich y otros (2000) desarrollan un estudio más reciente sobre el análisis de los factores de impacto en las revistas financieras más influyentes. En dicho estudio se tienen en cuenta los factores de impacto del Social Sciences Citation Index $(\text { SSCI o SCI })^{17}$, el cual es la medida más comúnmente utilizada para verificar la influencia de las revistas, en este caso las correspondientes al área de finanzas. De acuerdo con los reportes de dicho estudio, entre 1977 y 1990 -1998 el JFE lideró la lista de las revistas más importantes en el área financiera. El JOF, JFE y Review of Financial Studies (RFS) son, en la actualidad, las revistas de finanzas de mayor importancia. Se puede revisar, a través de las bases de datos digitales, el factor de impacto del número de consultas que tiene cada artículo.

Entre 1990 a 1996, la gran mayoría de los artículos se escribieron en la categoría de mercados financieros, 181 artículos del JOF, 138 de RFS y 78 de JFE. En las otras cuatro categorías, con excepción de las finanzas corporativas y públicas, la participación de artículos fue bastante precaria. Lo anterior sugiere que puede existir una tendencia a escribir más artículos sobre temas de mercados financieros que sobre instituciones financieras, dinero y tasas de interés, no solamente en estas tres revistas líderes, sino también en las demás que contribuyen al área financiera.

De acuerdo con la investigación desarrollada por Corrado y Ferris (1997), en la Tabla 2 se clasifican los artículos más utilizados para el diseño de seminarios de doctorado en Finanzas en las escuelas de negocios estadounidenses.

15. Estas categorías corresponden a los campos de investigación temáticos generales utilizados para escribir y publicar especialmente en el JOF; los artículos que no coincidan con estas categorías son clasificados dentro del grupo de finanzas generales.

16. Para ver el listado completo de todas las categorías véase Borokhovich y otros. (1994) pág. 720 a 721.

17. El SSCI se calcula sumando el número de veces que los artículos publicados en una revista en los años $(\mathrm{t}-1)$ y $(\mathrm{t}-2)$ son citados en el año $(\mathrm{t})$ y posteriormente se divide esa suma por el número total de artículos publicados en la revista en los años $(\mathrm{t}-1)$ y $(\mathrm{t}-2)$. Los factores de impacto demuestran ser un buen indicador de las influencias de largo plazo de las revistas. En bases de datos electrónicas como Ebsco, este número se encuentra entre paréntesis.

Para más información al respecto se puede consultar http://www.isinet.com. 


\section{Tabla 2. Artículos clásicos más utilizados en los programas de Doctorado en Finanzas.}

FAMA, Eugene (1970). "Efficient capital market: a review of theory and empirical work", The Journal of Finance, Vol. 25, No. 2, Papers and Proceedings of the Twenty-Eighth Annual Meeting of the American Finance Association New York, N.Y. December, 28-30, 1969. May, pp. 383-417.

GROSSMAN, Sanford J.; STIGLITZ, Joseph E. (1980). "On the Impossibility of Informationally Efficient Markets", The American Economic Review, Vol. 70, No. 3, June, pp. 393-408.

HARRIS, M.; RAVIV A. (1991). "The Theory of Capital Structure", Journal of Financial Economics, Vol. 46, No. 1, March, pp. 297-355.

JENSEN, Michael C. (1986). "Agency Costs of Free Cash Flow, Corporate Finance, and Takeovers", The American Economic Review, Vol. 76, No. 2, Papers and Proceedings of the Ninety-Eighth Annual Meeting of the American Economic Association. May, pp. 323-329.

JENSEN, M.; MECKLING W. (1976). "Theory of the firm: managerial behavior, agency costs and ownership structure", The Journal of Financial Economics, Vol. 3, October, pp. 305-360.

LELAND, H.; PYLE D. (1977). "Informational asymmetries, financial structure and financial intermediation", The Journal of Finance, Vol. 32, No. 2, May, pp. 371-387.

MODIGLIANI, Franco; MILLER, Merton H. (1958). "The Cost of Capital, Corporation Finance and the Theory of Investment", The American Economic Review, Vol. 48, No. 3, June, pp. 261-297.

ROSS, Stephen (1977). "The Determination of Financial Structure: the Incentive-signaling Approach", Bell Journal of Economics, Vol. 8, No. 1, March, pp. 24-40.

CHEN, Nai-Fu; ROLL, Richard; ROSS, Stephen A. (1986). "Economic Forces and the Stock Market", The Journal of Business, Vol. 59, No. 3, July, pp. 383-403.

ROLL, Richard; ROSS, Stephen A. (1980). "An Empirical Investigation of the Arbitrage Pricing Theory", The Journal of Finance, Vol. 35, №. 5, December, pp. 1073-1103.

FAMA, Eugene F.; FRENCH, Kenneth R. (1988). "Permanent and Temporary Components of Stock Prices", The Journal of Political Economy, Vol. 96, No. 2, April, pp. 246-273.

Fuente: Corrado y Ferris (1997).

De acuerdo con dicha investigación, 37\% de los artículos son considerados de los últimos cinco años debido a que ofrecen lo más reciente en teoría y metodología, mientras que el 30\% de los artículos son tomados para períodos de diez años en adelante. A este último grupo corresponden algunos de los artículos clásicos, los cuales están en el grupo de los artículos por excelencia más utilizados en los programas de doctorado en Finanzas; esto sugiere que la mayoría de programas de doctorado en finanzas en las diferentes universidades en el mundo utilizan esta bibliografía como un material obligatorio estándar para ser leído y discutido en los diferentes seminarios.

Aunque no todos los artículos reseñados en la Tabla 2 han sido merecedores de un premio Nobel de Economía, sí han sido contribuciones académicas importantes en cada uno de los respectivos campos de investigación de las finanzas ${ }^{18}$.

Con la gran cantidad de revistas académicas que existe en el mundo, lo primordial

18. Para ver la lista completa de estos artículos véase Corrado y Ferris (1997), pág. 2.101. Todos estos artículos, con excepción de los publicados por JFE, se encuentran en http://www.jstor.org. Actualmente bibliotecas como la de la Universidad del Valle, tienen acceso a los artículos del JFE, de los últimos cinco años, a través de http://www.sciencedirect.com. 
es identificar los líderes, dónde publican, en términos generales, las investigaciones más sobresalientes provenientes de las diferentes universidades y, especialmente, las investigaciones desarrolladas en las universidades más importantes del mundo. El nivel de evaluación por parte de los pares académicos de los artículos que aparecen publicados en estas revistas es bastante fuerte y en algunas ocasiones el proceso de evaluación puede demorar más de un año (en general, la mayoría de estas revistas sacan publicaciones cada dos meses). Igualmente estos artículos se encuentran indexados como artículos de máxima categoría.

En la Tabla 3 se puede ver la lista de las revistas académicas más importantes en el campo financiero. Especialmente las cuatro primeras revistas de esta lista son consideradas como líderes en publicar contribuciones académicas significativas, con desarrollos teóricos y empíricos importantes en el campo financiero y por lo tanto, las revistas académicas más competitivas y de mayor prestigio investigativo internacionalmente.

Al comparar el estudio de Corrado y Ferris (1997) con el de Borokhovich y otros, (2000) se observa que, de acuerdo con el factor de impacto del SSCI, y específicamente en la categoría de mercados financieros, el JOF sigue liderando la lista, seguido por el RFS, JFE y JFQA en segundo, tercero y cuarto lugar respectivamente ${ }^{19}$.

Tabla 3. Lista de las revistas académicas más importantes y de las organizaciones que las publican.

\begin{tabular}{|l|l|l|c|}
\hline SIGLA & \multicolumn{1}{|c|}{ TEXTO } & \multicolumn{1}{|c|}{ EDITORIAL } & PORCENTAJE \\
\hline JOF & Journal of Finance* & American Finance Association & $36,1 \%$ \\
\hline JFE & Journal of Financial Economics** & Rochester University & $27,9 \%$ \\
\hline JFQA & $\begin{array}{l}\text { Journal of Financial and } \\
\text { Quantitative Analysis }\end{array}$ & $\begin{array}{l}\text { University of Washington School } \\
\text { of Business Administration }\end{array}$ & $8,1 \%$ \\
\hline RFS & Review of Financial Studies & Oxford University Press & $5,0 \%$ \\
\hline JOB & Journal of Business & University of Chicago Press & $4,9 \%$ \\
\hline ECM & Econometrica & Econometric Society & $4,6 \%$ \\
\hline JPE & Journal of Political Economy & University of Chicago Press & $4,0 \%$ \\
\hline AER & American Economic Review & American Economic Association & $3,7 \%$ \\
\hline QJE & Quarterly Journal of Economics & MIT Press & $0,1 \%$ \\
\hline
\end{tabular}

Fuente: elaboración propia basada en: Corrado y Ferris (1997), http://www.jstor.org.

Nota: porcentajes tomados de 2.373 citas de 1.031 artículos (de acuerdo con Corrado y Ferris, 1997). Todas estas publicaciones, con excepción de JFE, se encuentran en la base de datos http://www.jstor.org.

* La serie de Shorter Papers se comenzó a publicar en el JOF a partir de 1989 y está dedicada a publicar notas, revisiones de texto, comentarios y artículos cortos. Para más información se puede consultar http://www.afajof.org.

** JFE no se encuentra en jstor. Para obtener información en línea al respecto se puede ver http://www.jfe.rochester. edu.

19. De acuerdo con charlas informales sostenidas en 2004 con profesores de finanzas de universidades estadounidenses visitadas por el autor, se pudo constatar que el orden de esta lista se sigue manteniendo en la actualidad. 
La Fundación

Nobel es la organización encargada de administrar las nominaciones $y$ tiene a su cargo las ceremonias depremiación el 10 de diciembre de cada año.

\section{RELEVANCIA DE LOS PREMIOS NOBEL DE ECONOMÍA EN LA INVESTIGACIÓN CIENTÍFICA ${ }^{20}$}

Los premios Nobel se comenzaron a otorgar en 1901 en Física, Medicina, Literatura y Paz, de acuerdo con los deseos de Alfred Nobel. Estos consisten en otorgar un reconocimiento a los descubrimientos, invenciones, desarrollos y trabajos más importantes, así como a los mejores trabajos realizados por la fraternidad de las naciones.

La Fundación Nobel es la organización encargada de administrar las nominaciones y tiene a su cargo las ceremonias de premiación el 10 de diciembre de cada año. En 1968 el Banco Central de Suecia, Sveriges Risksbank, instituyó el Premio Nobel de Economía y es la entidad oficial encargada de otorgar dicho premio. La Royal Swedish Academy es la autoridad encargada de otorgar los premios Nobel de Física y Química. Karolinska Institute en Estocolmo está encargado de otorgar el premio Nobel en Medicina o Fisiología. El Comité del Parlamento Noruego otorga el premio Nobel de la Paz. Y, la Swedish Academy otorga el premio Nobel en Literatura.

Inicialmente hubo cierto rechazo al premio Nobel de Economía; se cuestionaba si una ciencia social como la economía era lo suficientemente "científica" como para merecer un premio de la categoría de las "ciencias duras" como la Física y la Química. Después de algunos debates, en enero de 1969, el Gobierno Sueco codificó formalmente las reglas de este premio.

El premio Nobel es otorgado por desarrollos específicos en la ciencia, más que por científicos destacados individualmente; tiene presente las contribuciones permanentes en el período de vida de un científico, más que una contribución aislada, que realiza en un momento específico.

Lindbeck (1985), realiza una clasificación general en cinco grupos, de los varios tipos de premios Nobel de Economía otorgados hasta $1985^{21}$. Esta clasificación es la siguiente:

1. Teoría económica general. Samuelson, Arrow, Hicks y Debreu.

2. Contribuciones teóricas concernientes a aspectos o sectores específicos de la economía: Meade, Ohlin, Tobin, Stigler, Friedman, Lewis, Schultz y Simon.

3. Nuevos métodos de análisis económico, sus desarrollos y aplicaciones: Frish, Timbergen, Leontief, Stone, Koopmans y Kantarovich.

4. Más cercano a la investigación empírica "pura": Kuznets y Klein.

5. Pensamiento innovador no formalizado: Hayek y Myrdal.

20. Los premios Nobel de la Academia (The Royal Swedish Academy of Sciences) se ofrecen a seis disciplinas: Química, Física, Medicina, Economía, Literatura y Paz. En este orden de ideas, todos los avances en el área de las ciencias sociales, al menos hasta ahora, son laureados en la disciplina económica. El premio Nobel de Economía "Bank of Sweden Prize in Economic Sciences in Memory of Alfred Nobel”, se comenzó a otorgar anualmente, a mediados del mes de octubre, a partir del año de 1969. El procedimiento que sigue la Fundación Nobel cada año es el siguiente: la nominación de los candidatos a laurear se lleva a cabo a inicios de febrero, la selección a mediados de octubre (normalmente el 15 de octubre) y la premiación en diciembre, en la ciudad de Estocolmo (Suecia). Para más información se puede consultar http://www. nobel.se o http://www.nobelprize.org.

A través de jstor se puede conseguir la mayoría de los trabajos de investigación más importantes merecedores de un premio de la academia, así como los subsiguientes desarrollos en dichas líneas de investigación y la mayoría de publicaciones científicas de dichos autores.

21. Los premios Nobel de Economía otorgados hasta 2007 pueden también encajar en esta clasificación. Para ver la reseña completa de cada premio Nobel perteneciente a esta clasificación se puede consultar Lindbeck (1985), p. 39-45. 
Una buena razón para justificar el otorgamiento de los premios Nobel es “...mostrar, al menos una vez al año, al público general que existen otros 'héroes' en el mundo además de los atletas, artistas, estrellas de pop, políticos y otros grupos de entretenedores y tomadores de decisiones quienes dominan los reportajes en los medios masivos" (Lindbeck: 1985, 47). Lo anterior establece dos aspectos muy interesantes: por un lado los premios sirven para incentivar la alidad investigativa entre muchos docentes universitarios, y por otra, cuando los premios son otorgados, ponen al tanto al público general sobre los galardonados y sus contribuciones, e incluso inducen a leer algunos artículos o libros que de otra manera no habrían conocido.

La razón por la cual los premios Nobel de Economía se otorgan con un prolongado retraso (15 a 25 años) a partir de las contribuciones originales, es porque se considera importante esperar las críticas y las subsiguientes pruebas de la relevancia de las contribuciones, que para el caso de las ciencias sociales, toma más tiempo que en las "ciencias exactas", debido a que el comportamiento económico es más complejo y varía en el tiempo.

Un aspecto bastante importante a tener en cuenta es que los premios Nobel de Economía han reflejado varias características del análisis económico, especialmente de la segunda mitad del siglo XX. Sin embargo, éstos reflejan claramente el rol dominante de los Estados Unidos en la investigación económica. De los 39 premios Nobel de Economía otorgados hasta 2007 a 61 personas ${ }^{22}$, solamente 15 pertenecen o pertenecieron a universidades no estadounidenses en el momento de su nominación: Cambridge (3), Oslo (2), All Soul College Oxford (1), Stockholm (1), Netherland School of Economics (1), Academy of Ciences (1), Moscow (1), Freiburg (1), Trinity College (1), Bonn (1), Ecole Nationale Superieur des Mines de Paris (1), Israel (1) y Stockholm School of Economics (1). Especialmente en las dos últimas décadas existe una clara tendencia a la dominación casi total de las escuelas estadounidenses. Desde 1969 hasta 1978 se habían repartido ocho premios para escuelas estadounidenses e igual cantidad para escuelas no estadounidenses. A partir de 1979 hasta el 2007 se han repartido 38 y $1 / 2$ premios a escuelas estadounidenses y solamente 5 y $1 / 2$ premios (Stone, Allais, Haavelmo, Selten, Sen y Aumann ${ }^{23}$ ) a escuelas no estadounidenses. En la Tabla 4 se puede observar las cinco escuelas con la mayor cantidad de premios Nobel de Economía recibidos junto con sus

22. 2007 Leonid Hurwicz, Eric Maskin, Roger Myerson, 2006 Edmund Phelps, 2005 Robert J. Aumann, Thomas C. Schelling, 2004 Finn E. Kydland, Edward C. Prescott, 2003 Robert F. Engle, Clive W. Granger, 2002 Daniel Kahneman, Vernon L. Smith, 2001 George A. Akerlof, A. Michael Spence, Joseph E. Stiglitz, 2000 James J. Heckman, Daniel L. McFadden, 1999 Robert A. Mundell, 1998 Amartya Sen, 1997 Robert C. Merton, Myron S. Scholes, 1996 James A. Mirrlees, William Vickrey, 1995 Robert E. Lucas Jr., 1994 John C. Harsanyi, John F. Nash Jr., Reinhard Selten, 1993 Robert W. Fogel, Douglass C. North, 1992 Gary S. Becker, 1991 Ronald H. Coase, 1990 Harry M. Markowitz, Merton H. Miller, William F. Sharpe, 1989 Trygve Haavelmo, 1988 Maurice Allais, 1987 Robert M. Solow, 1986 James M. Buchanan Jr., 1985 Franco Modigliani, 1984 Richard Stone, 1983 Gerard Debreu, 1982 George J. Stigler, 1981 James Tobin, 1980 Lawrence R. Klein, 1979 Theodore W. Schultz, Sir Arthur Lewis, 1978 Herbert A. Simon, 1977 Bertil Ohlin, James E. Meade, 1976 Milton Friedman, 1975 Leonid Vitaliyevich Kantorovich, Tjalling C. Koopmans, 1974 Gunnar Myrdal, Friedrich August von Hayek, 1973 Wassily Leontief, 1972 John R. Hicks, Kenneth J. Arrow, 1971 Simon Kuznets, 1970 Paul A. Samuelson, 1969 Ragnar Frisch, Jan Tinbergen. Para ver una reseña de las diferentes contribuciones a la ciencia económica de todos los premios Nobel de Economía se puede consultar http://nobelprize.org/economics/laureates/ (se le da clic al autor respectivo y después al link Information for the Public).

23. El premio de Aumann se reparte así: 50\% escuela estadounidense, 50\% escuela israelí. 
respectivos laureados, los cuales eran profesores nombrados en dichas escuelas en el momento de su nominación.

\section{APORTE DIRECTO DE LOS PREMIOS NOBEL DE ECONOMÍA AL CAMPO FINANCIERO}

De los 61 premios Nobel de Economía entregados hasta 2007 , se podría mencionar prácticamente siete nominaciones otorgadas a 14 personas que han hecho contribuciones y aplicaciones directas al campo financiero y al área de Mercados Financieros; aunque existen obviamente otras nominaciones que también tienen aplicaciones, aunque menos directas, a dicho campo. Estas siete nominaciones se refieren específicamente a las principales áreas de las finanzas: fijación del precio de las opciones, finanzas corporativas, teoría del portafolio, fijación de precios de los activos, mercados financieros con información asimétrica, finanzas conductuales y análisis de riesgo de mercado.

Estas nominaciones son las siguientes:

1. 1981 Tobin James (1918-2002): Teoría de la inversión y de la selección de portafolio.

2. 1985 Modigliani Franco (1918-2003):
Análisis de los ahorros domésticos y los mercados financieros.

3. 1990 Markowitz Harry, Miller Merton (19232000), Sharpe William: Estudio de los mercados financieros y la toma de decisiones de inversión: Teoría de Portafolios, bases de la Teoría de las Finanzas Corporativas y Modelo de Fijación de Precios de Activos de Capital.

4. 1997 Merton Robert, Scholes Myron: Desarrollo de un método para determinar la fijación de precios de las opciones y otros derivados.

5. 2001 Akerlof George, Stiglitz Joseph, Spence Michael: Análisis de mercados con información asimétrica.

6. 2002 Kahneman Daniel, Smith Vernon: Integración de ideas de la psicología a la ciencia económica, respecto a los juicios humanos y a la toma de decisiones bajo incertidumbre; estudio de mecanismos de mercado alternativos.

7. 2003 Engle Robert, Granger Clive: Desarrollo de métodos para analizar las series de tiempo económicas con volatilidad cambiante en el tiempo (modelos ARCH); desarrollo de métodos para analizar las series de tiempo económicas con tendencias comunes (cointegración).

Tabla 4. Lista de las cinco universidades con la mayor cantidad de premios Nobel de Economía recibidos hasta 2007.

\begin{tabular}{|r|r|r|r|r|r|}
\hline & \multicolumn{1}{|c|}{ CHICAGO* } & \multicolumn{1}{c|}{ PRINCETON } & \multicolumn{1}{c|}{ BERKELEY } & \multicolumn{1}{c|}{ HARVARD } & \multicolumn{1}{c|}{ MIT } \\
\hline 1 & Myerson (2007) & Maskin (2007) & Akerlof (2001) & Merton (1997) & Solow (1987) \\
\hline 2 & Hekman (2000) & Kahneman (2002) & Mc. Fadden (2000) & Leontief (1973) & Modigliani (1985) \\
\hline 3 & Lucas (1995) & Nash (1994) & Hersanyi (1994) & Arrow (1972) & Samuelson (1970) \\
\hline 4 & Fogel (1993) & Lewis (1979) & Debreu (1983) & Kuznets (1971) & \\
\hline 5 & Becker (1992) & & & & \\
\hline 6 & Coase (1991) & & & & \\
\hline 7 & Miller (1990) & & & & \\
\hline 8 & Stigler (1982) & & & \\
\hline 9 & Schultz (1979) & & & & \\
\hline 10 & Friedman (1976) & & & & \\
\hline
\end{tabular}

Fuente: elaboración propia.

Nota: todos los economistas laureados pertenecen o pertenecieron (para los que ya han fallecido) a dichas instituciones universitarias, en calidad de profesores nombrados en el momento de su nominación. De acuerdo con las reglas de la Real Academia Sueca, los premios se pueden repartir máximo entre tres personas y las proporciones son: 100\%; $50 \%, 50 \% ; 33 \%, 33 \%, 33 \%$ y $50 \%, 25 \%, 25 \%$. Actualmente el premio corresponde a una suma de US $\$ 10.000 .000$ repartidos entre los laureados respectivos. 
* De los 61 premios Nobel de Economía otorgados hasta 2007 Chicago tiene el mérito de tener 24 premios Nobel de Economía que en algún momento de su carrera pertenecieron a la Universidad de Chicago en calidad de estudiantes, profesores o investigadores; en la actualidad (2007) sólo hay siete premios Nobel de Economía como profesores nombrados. Por otro lado, Chicago es la universidad que más premios Nobel ha obtenido: en total son 77 premios Nobel en todas las disciplinas hasta 2003; para más información se puede consultar http://www.uchicago.edu.

Las investigaciones merecedoras de dichos premios fueron publicadas originalmente en varias de las revistas académicas referenciadas en el presente artículo. La mayoría de éstas son de un contenido cuantitativo, metodológico y teórico bastante complejo, especialmente por la dificultad de los modelos matemáticos utilizados, de caracter econométricos y microeconómicos, y el desarrollo de teoremas y teorías para sustentar dichas investigaciones; por consiguiente, tienen un grado de dificultad de lectura (se debe tener como mínimo unas fuertes bases matemáticas, estadísticas y econométricas previas para analizar a plenitud dichas publicaciones); sin embargo, es precisamente eso lo que los hace tan atractivos para tenerlos como material de referencia obligatorio en el campo de la investigación académica científica. Véanse dichos artículos en la Tabla 5.

\section{Tabla 5. Artículos más importantes merecedores de un premio Nobel de Economía, con aportes al campo financiero.}

ENGLE, Robert F. (1982). "Autoregressive conditional heteroscedasticity with estimates of the variance of United Kingdom inflation", Econométrica, Vol. 50, No. 4, pp. 987-1008.

KAHNEMAN, Daniel; Tversky Amos (1979). "Prospect Theory: An Analysis of Decision under Risk", Econométrica, Vol. 47, No. 2. March, pp. 263-293.

VERNON, L. Smith (1962). "An Experimental Study of Competitive Market Behavior", The Journal of Political Economy, Vol. 70, No. 2. April, pp. 111-137.

AKERLOF, George A. (1970). "The market of "lemons": qualitative uncertainty and the market mechanism", Quarterly Journal of Economics, Vol. 84, No. 3. August, pp. 488-500.

SPENCE, Michael (1973). "Job Market Signaling”, The Quarterly Journal of Economics, Vol. 87, No. 3, August, pp. 355-374

GROSSMAN, Sanford J.; STIGLITZ, Joseph E. (1980). "On the Impossibility of Informationally Efficient Markets", The American Economic Review, Vol. 70, No. 3. June, pp. 393-408.

MERTON, Robert C. (1973). "Theory of Rational Option Pricing", The Bell Journal of Economics and Management Science, Vol. 4, No. 1. Spring, pp. 141-183.

BLACK, Fischer; SCHOLES, Myron (1973). "The Pricing of Options and Corporate Liabilities", The Journal of Political Economy, Vol. 81, No. 3. May-June, pp. 637-654.

MARKOWITZ, Harry (1952). "Portfolio selection", The Journal of Finance, Vol. 7, No. 1, March, pp. 77-91.

SHARPE, William (1964). "Capital asset prices: a theory of market equilibrium under conditions of risk", Journal of Finance, Vol. 19, September, pp. 425-442.

MERTON, H. Miller; FRANCO, Modigliani (1961). "Dividend Policy, Growth, and the Valuation of Shares", The Journal of Business, Vol. 34, No. 4. October, pp. 411-433.

Fuente: elaboración propia (algunos autores tienen dos o más artículos que también han sido galardonados). 
Aunque estas investigaciones fueron realizadas casi dos décadas antes del otorgamiento del premio Nobel respectivo, produjeron, en su época, un cambio de paradigma, con ideas revolucionarias que marcarían el desarrollo de nuevas y fuertes líneas de investigación. Con el tiempo estas investigaciones han sido reescritas en libros, con un lenguaje y metodología sencillos.

\section{SÍNTESIS DE LA EVOLUCIÓN DEL PENSAMIENTO ECONÓMICO Y LA ECONOMÍA FINANCIERA}

El pensamiento económico ha evolucionado notablemente desde la escuela clásica hasta la dinámica caótica y los campos afines. Los conceptos de equilibrio, linealidad y eficiencia de mercado se han ido transformando en no-linealidad, no-equilibrio e ineficiencia (véase la Tabla 6). De esta manera, los cuerpos teóricos de las escuelas económicas más antiguas (clásica, neoclásica, keynesiana, postkeynesiana, monetarista) ya no son tan útiles para explicar el comportamiento actual de los mercados y de los agentes que participan en ellos, puesto que están basados en supuestos de equilibrio y linealidad.

Tabla 6. Síntesis de la evolución de las principales escuelas de pensamiento económico y sus autores ${ }^{24}$

\begin{tabular}{|l|l|}
\hline Mercantilismo [1500-1750], PETTY (1675): diversas corrientes de la economía. \\
\hline Fisiocracia [1500-1750], QUESNAY (1758): algunos principios económicos. \\
\hline Clásica [1750-1870], enfoque global. Nace la economía como ciencia. \\
\hline$\bullet$ & SMITH (1776): ley del orden natural, neutralidad del Estado, laissez-faire. \\
\hline$\bullet$ & RICARDO (1817): armonía limitada y parcial del orden natural. \\
\hline$\bullet$ & MALTHUS (1820): armonía limitada y parcial del orden natural. \\
\hline Neoclásica [1870-1930], alejamiento de la realidad, enfoque equilibrista y estático. \\
\hline$\bullet$ & WALRAS (1874): Teoría General como puente entre micro y macro. \\
\hline$\bullet$ & MENGER (1875): determinismo mecanicista. \\
\hline$\bullet$ & MARSHALL (1890): análisis estático. \\
\hline$\bullet$ & PARETO (1906): equilibrio. \\
\hline Keynesianismo [1930-1974], revolución keynesiana (síntesis keynesiana - neoclásica). \\
\hline$\bullet$ & KEYNES (1936): incertidumbre. \\
\hline$\bullet$ & HICKS - HANSEN (1938 - 1953): sociologismo. \\
\hline$\bullet$ & SRAFFA (1960): desequilibrio. \\
\hline Postkeynesianismo [1938-1974]. \\
\hline$\bullet$ & KALEKY (1938 - 1954): macroeconomía. \\
\hline$\bullet$ & ROBINSON (1962): estática comparativa. \\
\hline$\bullet$ & PASINETTI (1974): estática comparativa. \\
\hline Monetarismo [1953 - 1974]: Equilibrio General. \\
\hline$\bullet$ & FRIEDMAN (1953): equilibrio. \\
\hline
\end{tabular}

24. Las fechas entre corchetes corresponden al período de vigencia de cada escuela económica en particular y las fechas entre paréntesis corresponden al año de publicación de la obra más representativa de cada autor (se listan los más relevantes). 


\begin{tabular}{|ll|}
\hline$\bullet$ & PHELPS (1967): automatismo. \\
\hline$\bullet$ & ARROW (1954) (1971): desarrollo de la Teoría Económica General. \\
\hline$\bullet$ & DEBREU (1959): Teoremas Punto Fijo. \\
\hline$\bullet$ & HAHN (1971): agotamiento progresivo. \\
\hline Teoría de los Desequilibrios [1977 -1984]. \\
\hline$\bullet$ & MALINVAUD (1977, 1980): equilibrio no-walrrasiano. \\
\hline$\bullet$ & BENASSY (1984): síntesis. \\
\hline Nueva Macroeconomía Clásica [1981 - 1986]: conducta optimizadora intertemporal. \\
\hline$\bullet$ & LUCAS, BARRO (1981 - 1986): Teoría de las Expectativas Racionales. \\
\hline$\bullet$ & MAC CALLUM (1986): determinismo reduccionista, restricciones presupuestarias. \\
\hline$\bullet$ & KYDLAND - PRESCOTT (1986): "agentes representativos". \\
\hline Nueva Economía Keynesiana [1989 - 1991]: fallas de mercado, competencia imperfecta. \\
\hline$\bullet$ & BLANCHARD (1989): fundamentación microeconómica. \\
\hline$\bullet$ & MANKIW (1991): fallas del mercado. \\
\hline Teoría de Juegos (Juegos Dinámicos) [1990 - 1992]: aportes del Equilibrio Nash. \\
\hline$\bullet$ & KREPS (1990): nuevos desarrollos. \\
\hline$\bullet$ & GIBBONS (1992) nuevos desarrollos. \\
\hline Dinámica Caótica y campos afines [1990 - al presente]. \\
\hline$\bullet$ & GRANDMONT (1985): endogeneidad. \\
\hline$\bullet$ & ARROW (1988): no linealidad. \\
\hline$\bullet$ & GOODWIN (1990): no - equilibrio. \\
\hline$\bullet$ & ROMER (1986, 1990): endogeneidad. \\
\hline$\bullet$ & LORENZ (1989): sistemas jerárquicos. \\
\hline$\bullet$ & DAY (1994): complejidad. \\
\hline$\bullet$ & SIMON (1996): complejidad. \\
\hline$\bullet$ & KRUGMAN (1996): autorganización. \\
\hline
\end{tabular}

Fuente: síntesis del autor y Díaz $(2000,46)$.

El hecho de que los premios Nobel de Economía tengan una relación importante con el desarrollo de la ciencia económica, no quiere decir que la ciencia económica como tal, se limite sólo a las aportaciones de los premios Nobel de Economía. Aunque, hasta ahora, solamente siete exponentes de algunas de las escuelas de pensamiento económico, presentados en la Tabla 6, han recibido un premios Nobel de Economía (Hicks, Friedman, Arrow, Debreu, Kydland, Prescott y Phelps), no significa que las escuelas de pensamiento económico que no hayan recibido un premio Nobel de Economía no sean lo suficientemente científicas.

La ciencia -Física-Matemática- ${ }^{25}$ ha evolucionado con el tiempo, y de la misma manera lo ha hecho la ciencia económica. Durante la segunda mitad del siglo XX, desde el punto de vista financiero, se han dado cambios relevantes en la economía como la desaparición del patrón oro, el desarrollo del dinero electrónico, la expansión de los euromercados, la globalización financiera, la proliferación de las crisis bursátiles, el desarrollo del $e$-trading, entre otros, que han cambiado

25. La Física-Matemática es la ciencia más "pura” que existe y con el tiempo ha marcado el derrotero de las demás ciencias. Esta ha evolucionado desde la Física Clásica (equilibrio) hasta la Física Cuántica (no-equilibrio). 
de una manera importante la forma tradicional de estudiar la economía y las finanzas. Para abordar este componente financiero en el contexto de la ciencia económica surgió la Economía Financiera; ésta se ocupa de estudiar uno de los tres grandes mercados de la economía: el mercado de activos financieros.

La Economía Financiera tiene sus orígenes en la Teoría Financiera Clásica, en 1900, la cual se ocupaba principalmente de estudiar los desarrollos de los Fundamentalistas y los Técnicos (véase la Tabla 7); sin embargo adquiere su nombre como tal a partir de la década de 1950 cuando los Cuantitativos comienzan a aplicar fuertemente la econometría en la medición de las variables económicas cuantitativas. Para esa época surgió la Teoría Moderna del Portafolio (TMP) que se desarrolló a partir de la fusión entre la Hipótesis de Eficiencia de los Mercados (HEM) ${ }^{26}$ y el Modelo de Valuación de Activos de Capital. La TMP trata sobre la toma de decisiones de inversión en condiciones de equilibrio y eficiencia de mercado y está dedicada a estudiar el portafolio de inversiones financieras. Por otro lado, surgió posteriormente la Teoría del Comportamiento Financiero (TCF), la cual estudia la toma de decisiones de inversión en condiciones de no-equilibrio e ineficiencia de mercado y está dedicada a la influencia de la psicología en el comportamiento de los profesionales en finanzas.

Al igual que en la evolución de la ciencia económica, desde lo lineal a lo nolineal, la TMP y la TCF son opuestas en sus cuerpos teóricos. Sin embargo la TMP arraiga en los académicos que estudian la Economía Financiera, entre otras razones porque, hasta el presente, seis de sus fundadores han recibido un premio Nobel de Economía (Tobin, Modigliani, Markowitz, Miller, Sharpe, Merton y Scholes, véase nuevamente la Tabla 5) ${ }^{27}$.

26. Esta hipótesis, en su versión débil, dio origen a la Teoría del Recorrido Aleatorio, que plantea que los sucesivos cambios en los precios de las acciones son serialmente independientes.

27. Sin embargo, varios de estos premios Nobel y otros Cuantitativos partidarios de la TMP, han sido criticados por Kurtzman (1993), entre varios autores, por ayudar a desestabilizar el sistema financiero internacional al crear ecuaciones matemáticas que determinarían los precios del mercado las cuales, con el tiempo, dejaron de dar resultados y crearon alta volatilidad y crisis en los mercados financieros mundiales (véase el caso del fondo de cubrimiento de riesgo LTCM). Al respecto Kurtzman (1993) comenta que estos académicos, especialmente matemáticos, economistas e ingenieros, a partir de la década de 1970, empezaron a entrar al sector privado a diseñar las estrategias de operación de las grandes firmas de corretaje. Ellos escribieron software para las megacomputadoras de las grandes firmas, además algunos empezaron a operar como agente de valores. Estos Cuantitativos académicos salieron de universidades como MIT, Harvard, Chicago, Berkeley, donde desarrollaron sus teorías y fueron contratados con salarios millonarios por grandes firmas como Salomón Brothers, UBS, Goldman Sachs, Leheman Brothers, JP Morgan, para que desarrollaran la estrategia cuantitativa con la cual la firma iba a hacer dinero. Como ejemplo se menciona el caso de Fisher Black, un matemático con un Ph.D. de Harvard, que para 1983 se ganaba US \$43.000 al año como profesor de matemáticas en el MIT. En 1984 fue contratado por Goldman Sachs y en 1986 pasó a ser socio de la firma y su sueldo anual ascendió a más de US \$1 millón al año (Kurtzman 1993, 23). Black ayudó a desarrollar el modelo Black-Scholes para determinar el valor de las opciones (véase el artículo donde se presentó esta fórmula, en la Tabla 5). A partir de la creación de la fórmula de dicho modelo, se creó una industria financiera completa que coincidió con la apertura de la Chicago Board Options Exchange, la cual fuela primera bolsa de opciones debidamente organizada en el mundo, y a partir de entonces comenzaron a surgir bolsas de opciones en las grandes plazas bursátiles mundiales: Tokyo, Londres, Sydney, Toronto, París. El problema fue que se empezaron a inundar las economías mundiales con productos financieros totalmente etéreos, sin referentes intrínsecos, como las opciones sobre tipos de interés u opciones sobre índices bursátiles, los cuales eran valorados de acuerdo con las fórmulas desarrolladas por Black, Merton, Sharpe (modelo CAPM), y con éstos se constituían en portafolios óptimos de acuerdo con las fórmulas desarrolladas por Markowitz, en un ambiente de libre competencia propuesto por los monetaristas (Friedman). Este modo de operación ha contribuido a generar grandes ataques especulativos en los mercados financieros rápidamente 
Tabla 7. Síntesis de la Economía Financiera y sus principales autores.

Teoría Financiera Clásica [1900 - 1950]. Especulación en los mercados, comportamiento corporativo.

- BACHELIER (1900): aplicación de métodos estadísticos para analizar los mercados financieros (Cuantitativos).

- GRAHAM y DOOD (1934): aplicación del Análisis Fundamental a la economía (Fundamentalistas).

- EDWARDS y MAGEE (1936): análisis de las tendencias de los mercados financieros (Técnicos).

Hipótesis de la Eficiencia de los Mercados [1950 - 1980]. Eficiencia de mercado, linealidad, normalidad.

- MODIGLIANI y MILLER (1961): política de dividendos y valuación de acciones.

- COOTNER (1964): carácter aleatorio del mercado bursátil.

- OSBORNE (1964): precios de las acciones son independientes (bases de la Teoría del Recorrido Aleatorio).

- FAMA (1965): mercado bursátil es un "juego limpio", la información no se puede utilizar para obtener ganancias.

- FAMA (1970): mercado de capitales es eficiente en su forma fuerte.

- LORIE y HAMILTON (1973): desacuerdo con los Fundamentalistas (surge la Hipótesis Semifuerte de Mercado).

Teoría Moderna del Portafolio [1950 - 1980]*. Equilibrio, normalidad y eficiencia de mercado.

- TOBIN (1950): preferencias inversionistas y posibilidad de combinar el rendimiento y el riesgo de mercado.

- MARKOWITZ (1952): Teoría de Selección del Portafolio financiero; rendimientos siguen un recorrido aleatorio.

- SHARPE (1964): modelo CAPM (Capital Asset Pricing Model).

- LINTNER (1965): a partir del modelo CAPM propuesto por Sharpe trata el problema del presupuesto de capital.

- MOSIN (1966): a partir del modelo CAPM, resuelve el problema de la falta de precisión en las condiciones de equilibrio.

- MERTON (1973): valuación de opciones desde el punto de vista de la racionalidad económica.

- BLACK y SCHOLES (1973): modelo de valuación de opciones en condiciones de equilibrio.

- ROSS (1976): desarrollo del modelo APT (Arbitrage Pricing Theory).

- ROSS y ROLL (1980): desarrollo APT en equilibrio de mercado, teniendo en cuenta la variabilidad común de los activos.

Teoría del Comportamiento Financiero [1970 - presente]. Mercados ineficientes, no-equilibrio, sesgos heurísticos.

- SLOVIC (1969): análisis proceso de decisión de los corredores de acciones.

- SLOVIC (1972): estudio psicológico de la toma de decisiones de inversión aplicado a los inversionistas.

- KAHANEMAN y TVERSKY (1974): existencia de errores guiados por heurísticos en la toma de decisiones bajo riesgo.

- KAHANEMAN y TVERSKY (1979): Teoría de las Perspectivas, en la cual se expone la dependencia del contexto.

- SHEFRIN y STATMAN (1978): preferencia de los dividendos en efectivo por parte de los inversionistas.

- DeBONDT y THALER (1985): existencia de la sobrerreacción de los inversionistas a las buenas y malas noticias.

- SHEFRIN y STATMAN (1985): dependencia del contexto a la realización de pérdidas en condiciones reales de mercado.

- TVERSKY y otros (1997): el Efecto Miopía y la aversión al riesgo en la toma de decisiones bajo incertidumbre.

- SHEFRIN (2000): sistematización y acuñación de la TCF y comprobación de sus supuestos en el contexto actual.

Fuente: elaboración propia.

Para una exposición más detallada véase Monroy (2005, 18-41).

*La TMP resurge a partir de la investigación desarrollada por Fama Eugene (1998): "Market efficiency, long-term returns and behavioral finance", Journal of Financial Economics, Vol 49, pp. 283-306, en la cual unifica la HEM y el CAPM como un único concepto y donde todas las imperfecciones de mercado son tomadas como anomalías.

las economías mundiales como ha sido el caso del Crash de 1987, la Crisis Tequila de 1994, la Crisis Asiática de 1997, la Crisis Rusa de 1998, el colapso de LTCM en 1998 (fondo de cobertura que tenía a dos premios Nobel como socios fundadores: Scholes y Merton). (Aquí no se discute en absoluto la falta de nivel académico de estas universidades y los respectivos autores, sino su sesgo ideológico neoliberal). 
Por otro lado, el estudio de los mercados financieros (área principal de las Finanzas), consiste en la aplicación de varias teorías y técnicas que involucran tanto desarrollos académicos como comerciales, los cuales provienen del análisis cuantitativo, fundamental y técnico, y que tienen la finalidad de controlar todos los riesgos inherentes al mercado y al mismo tiempo tratan de hacer predicciones de las tendencias financieras-, también han mostrado una fuerte evolución desde los enfoques lineales a los no-lineales, es decir, desde lo mecanístico hasta la inteligencia artificial (véase la Tabla 8). Los desarrollos más importantes se han hecho en relación con el tema de inversiones principalmente alrededor de los mercado bursátiles y de los mercados derivados, los cuales son dos de los componentes principales de los Mercados Financieros.

Tabla 8. Síntesis de las escuelas y los principales autores que estudian el control de riesgos y la predicción de tendencias de los mercados financieros.

\begin{tabular}{|c|c|}
\hline \multicolumn{2}{|r|}{ MECANÍSTICO } \\
\hline Comportamiento cíclico & $\begin{array}{l}\text { D. Dewey y Og (1973): estudio de los ciclos. } \\
\text { Kondratieff (1984): gran Ola de Kondratieff. }\end{array}$ \\
\hline Generación de ruido. & $\begin{array}{l}\text { Cootner (1964): Teoría Financiera Clásica, Teoría del Recorrido Aleatorio. } \\
\text { Markowitz (1952): pilares de la Teoría Moderna del Portafolio. }\end{array}$ \\
\hline \multicolumn{2}{|r|}{ EMERGENTE } \\
\hline Psicología bursátil. & $\begin{array}{l}\text { Kahneman y Tversky (1979): Teoría de las Perspectivas. } \\
\text { Shefrin (2000): sistematización de la Teoría del Comportamiento Financiero. }\end{array}$ \\
\hline \multicolumn{2}{|r|}{ COMPORTAMIENTO FRACTAL } \\
\hline Teoría Dow. & Rhea (1932): bases teóricas de la Teoría Dow. \\
\hline \multicolumn{2}{|c|}{ Herramientas Técnicas. Murphy (1999): volumen, líneas de tendencia, soportes, resistencias. } \\
\hline Chartismo Clásico. & Edwards y Magee (1966): patrones de reversa, continuación y mixtos. \\
\hline Velas Japonesas. & Nison (1991): patrones Doji, Marobozu, Tasuki, Harami. \\
\hline Ondas de Elliott. & Frost y Prechter (1998): patrones, razones y tiempo en las ondas. \\
\hline \multirow[t]{2}{*}{ Matemática Fibonacci. } & Basin (1963): secuencia de la serie Fibonacci y propiedades matemáticas. \\
\hline & TEORÍA DEL CAOS \\
\hline Física no lineal. & $\begin{array}{l}\text { Lorenz (1963): efecto mariposa. } \\
\text { Mandelbrot (1961): funciones estadísticas paretianas. }\end{array}$ \\
\hline \multirow[t]{2}{*}{ Economía. } & $\begin{array}{l}\text { Peters (1996): Hipótesis Fractal y aplicación a la bolsa. } \\
\text { Díaz (2000): aplicación a la economía. }\end{array}$ \\
\hline & ANÁLISIS TÉCNICO MATEMÁTICO \\
\hline Valoración de riesgo. & $\begin{array}{l}\text { Jorion (1996): modelos de Value at Risk (VaR). } \\
\text { Engle (1982): modelos ARCH. }\end{array}$ \\
\hline Indicadores técnicos. & Colby y Myers (1988): OBV, medias móviles, Momentum. \\
\hline Osciladores técnicos. & Brown (1999): Estochastics (\%K), RSI, MACD, Composite Index. \\
\hline \multicolumn{2}{|c|}{ Seguidores de tendencia. Wilder (1978): ADX, Parabolic SAR. } \\
\hline \multirow[t]{2}{*}{ Sistemas operacionales. } & Chande (1997): desarrollo de sistemas operacionales discrecionales, RAVI. \\
\hline & INTELIGENCIA ARTIFICIAL \\
\hline Redes neuronales. & Refenes (1993): redes neuronales artificiales aplicadas a bolsa. \\
\hline Algoritmos genéticos. & Bauer (1994): aplicación del comportamiento de evolución-adaptación a la bolsa. \\
\hline \multirow{2}{*}{$\begin{array}{l}\text { Lógica difusa. } \\
\text { Aprendizaje máquina. }\end{array}$} & Chorafas (1994): aplicación de la lógica trivalente a las cotizaciones de bolsa. \\
\hline & Ruggiero (1997): aplicación a bolsa. \\
\hline
\end{tabular}

Fuente: Monroy (2005). 
Respecto a la información asimétrica en los mercados financieros Akerlof (1970) elabora una aplicación del Principio de los Cacharros $^{28}$ (Lemons Principle) al mercado de crédito en los países subdesarrollados y expone el caso de la India donde el prestamista local prácticamente extorsiona a sus clientes con tasas de interés supremamente altas. Mientras los grandes bancos en las ciudades centrales tienen tasas de interés preferenciales de $6 \%, 8 \%$ y $10 \%$, el prestamista local, exige tasas de $15 \%$, 25\% e inclusive del 50\% (Akerlof: 1970, 498). La situación aquí es que el crédito es concedido solamente donde el prestamista tiene medios fáciles para recuperarlo o tiene conocimiento personal de las características del tenedor del préstamo, de lo contrario, para obtener un préstamo hay que someterse a las altas tasas usurarias de los prestamistas locales, sin conocer o sin tener derecho a las tasas normales del sistema financiero, generando completamente una situación de manejo de información asimétrica.

Stiglitz y Grossman (1980) plantean que la afirmación de que todos los mercados están siempre en equilibrio y el arbitraje es realizado en perfectas condiciones, es inconsistente cuando el arbitraje es costoso. Ellos proponen un modelo donde exista un grado de equilibrio del desequilibrio; es decir, los precios reflejan los precios de los individuos informados (arbitrageurs), pero sólo parcialmente, de tal manera que aquellos que gastan recursos para obtener información reciben una compensación. Por lo tanto, qué tan informativo es el sistema de pre- cios depende del número de individuos que están informados, sin embargo, el número de individuos que están informados es por sí misma una variable endógena del modelo (Stiglitz y Grossman: 1980, 393).

Este modelo es el más sencillo, en el cual los precios desarrollan un rol bien articulado en transmitir información desde los informados hacia los no informados. De esta manera, cuando los individuos informados observan alguna información de que los rendimientos de una acción van a estar altos, demandan su precio al alza, y a la inversa, cuando ellos observan información de que los rendimientos de la acción van a estar bajos. Por consiguiente, el sistema de precios hace públicamente disponible la información obtenida por los individuos informados a los no informados. Sin embargo, en general, esto sucede de una manera imperfecta y por tanto, una situación de equilibrio no podría existir.

Stiglitz y Grossman (1980) elaboran un modelo matemático y macroeconómico con seis teoremas principales y concluyen que, en general, el sistema de precios no revela toda la información acerca del "valor verdadero" de los activos de riesgo. De tal forma que la única manera como los inversionistas ${ }^{29}$ pueden generar una ganancia en su actividad de recolección de información es si ellos pueden utilizar su información para tomar posiciones en el mercado "mejores" que las posiciones de los inversionistas no informados (Stiglitz y Grossman: 1980, 404). Por lo tanto, si los precios en cualquier momento reflejan completamente toda la información disponible, tal como lo plan-

28. El Principio de los Cacharros plantea, en síntesis, que a la hora de comprar un vehículo usado, normalmente el vendedor tiene más información acerca del estado y la calidad del vehículo que el comprador que lo va a adquirir. Esto simplemente demuestra una situación de manejo de información asimétrica.

29. Traders (corredores, agentes de valores) en el original. 
Elprimer y segundo problema muestran que la gente le da un mayor peso a los resultados que son considerados seguros, con respecto a los resultados que son considerados probables. tean los teóricos de la HEM, entonces los inversionistas informados no podrían generar una ganancia en el uso de su información. Stiglitz y Grossman (1980) demuestran que cuando la hipótesis de mercado eficiente es verdadera y la información tiene un costo, dejan de existir los mercados competitivos. Cuando esto pasa, cada inversionista informado siente que puede parar de pagar por información y negociar igual a como lo haría un inversionista que no paga nada por la información. Al parecer todos los inversionistas informados piensan de esta manera, por tanto, tener una fracción de individuos informados no es una situación de equilibrio, y no tener a nadie informado tampoco es una situación de equilibrio, porque cada inversionista, al tomar el precio como dado, piensa que hay ganancias por realizar al ser informado. Los teóricos de la HEM parecen estar conscientes de que la información sin costo es una condición suficiente para que los precios reflejen completamente toda la información disponible; sin embargo, estos no son conscientes de que es una condición necesaria. De acuerdo con el modelo propuesto se intenta redefinir la noción de "eficiencia de mercado"y no destruirla (Stiglitz y Grossman: 1980, 404).

Kahneman y Tversky (1979) desarrollan la Teoría de las Perspectivas, base de la TCF, y para el año 2002 Kahneman recibió el premio Nobel de Economía por estos desarrollos, con lo cual se le dio un mayor peso científico a la TCF, validando así las críticas contra algunos supuestos de la TMP. Kahneman y Tversky presentaron una crítica a la Teoría de la Utilidad Esperada (TUE), la cual, hasta entonces, venía dominando el análisis de la toma de decisiones bajo condi- ciones de riesgo y desarrollaron un modelo alternativo llamado Teoría de las Perspectivas o Posibilidades, que básicamente muestra que las elecciones entre posibilidades riesgosas exhiben efectos dominantes que son inconsistentes con los principios de la TUE.

Las demostraciones empíricas que realizan Kahneman y Tversky (1979) para probar la violación de dichos principios están basadas en las respuestas de estudiantes universitarios y miembros de la facultad a problemas de elección hipotética. En dicha investigación se presentan y discuten 14 problemas.

El primer y segundo problema muestran que la gente le da un mayor peso a los resultados que son considerados seguros, con respecto a los resultados que son considerados probables. Esto es lo que Kahneman y Tversky $(1979,265)$ llamaron "Efecto Seguridad" (Certainty Effect):

PROBLEMA 1: escoja entre:

A: $\quad \$ 2.500$ con probabilidad de $33 \%$, $\$ 2.400$ con probabilidad de $66 \%$ $\$ 0$ con probabilidad de $1 \%$

$$
\text { [18\%] }
$$

B: $\quad \$ 2.400$ con seguridad

$$
\text { [82\%] } \quad \mathrm{N}=72
$$

PROBLEMA 2: escoja entre:

C: $\$ 2.500$ con probabilidad de 33\%, $\$ 0$ con probabilidad de $67 \%$, [83\%]

D: $\quad \$ 2.400$ con probabilidad de $34 \%$ $\$ 0$ con probabilidad de $66 \%$

$$
\text { [17\%] } \quad \mathrm{N}=72
$$

En el Problema 1, 82\% de los individuos escogen la opción B, y 83\% escogen la opción

30. El porcentaje de participantes que escoge cada opción está dentro de corchetes y $\mathrm{N}$ es el número de participantes totales que responde cada prueba. Los resultados monetarios están denominados en divisa israelí. Como dato de referencia, para aquella época \$ 3.000 libras israelíes (hoy shekel) equivalían a un salario promedio mensual para una familia corriente. 
Cen el Problema 2. Este patrón de referencias viola la TUE, en la manera originalmente propuesta por el economista, premio Nobel, Maurice Allais en un artículo publicado en 1953. De acuerdo con esta exposición $U$ $(0)=0$, entonces la primera preferencia $(B)$ implica que $U(2.400)>33 \% U(2.500)+$ $66 \% U(2.400)$ o $34 \% U(2.400)>33 \% U$ (2.500), mientras que la segunda preferencia (C) implica la desigualdad invertida. El Problema 2 plantea una gran reducción en la escogencia cuando es alterado el carácter de la perspectiva de segura a probable. Lo anterior quiere decir que los individuos prefieren una mayor cantidad de dinero (\$2.500 en vez de \$2.400), sin importar que la mayor cantidad de dinero tenga una probabilidad de obtención menor que la menor cantidad de dinero $(33 \%<34 \%)$ y los individuos erróneamente prefieren la opción (C), sin reparar en que tienen la mayor probabilidad de no ganar nada (\$0).

En esta investigación Kahneman y Tversky (1979) también desarrollan y discuten el Efecto Reflexión, el Efecto Aislamiento y la Función de Valor. Con lo anterior demuestran que existe una dependencia del contexto en la toma de decisiones financieras, lo cual establece que los mercados financieros son ineficientes.

Recientemente, Frankfurter y otros (2004) desarrollan una investigación en la que muestran el cambio de las Finanzas Modernas (TMP) a la Economía Financiera (TCF), centrándose en la idea de la forma en que realmente toma las decisiones el homo economicus. Esta investigación hace un recorrido teórico de ambos enfoques y sustenta bien las críticas a los supuestos de la TMP originadas por algunos de sus fundadores. Igualmente realiza unas críticas al uso de la matemática como instrumento de dominio en las finanzas, argumentando que aunque ha arraigado en la comunidad académica por diez razones fundamentales (véase Frankfurter y otros (2004), 464), esta no resuelve totalmente el problema del comportamiento del ser humano y, por consiguiente, la toma de decisiones de inversión. Frankfurter y otros (2004) concluyen que la TCF no debería ser considerada como un modelo alternativo a la TMP, sino como un desarrollo teórico contrario a la TMP que explica mejor el comportamiento del ser humano (homo economicus) en la toma de decisiones de inversión, el cual debe seguirse investigando.

Por otro lado, respecto a la no-normalidad delas series de tiempo financieras, Engle (1982) desarrolla los modelos ARCH ${ }^{31}$. En esta clase de modelos la varianza de una serie de datos depende dela información pasada. Engle desarrolla una aplicación en teoría monetaria y en teoría financiera. Partiendo de la visión de la TMP de que los portafolios de los activos financieros son considerados como medias y varianzas esperadas de las tasas de retorno, cualquier cambio en la demanda de los activos debe estar asociado con cambios en la media y la varianza esperadas de las tasas de retorno. Si se asume que la media sigue un modelo de regresión estándar o de serie de tiempo, la varianza es inmediatamente forzada a ser constante en el tiempo, lo cual no sería algo apropiado, y además, el uso de variables exógenas para explicar los cambios en la varianza usualmente no es adecuado (Engle: 1982, 989).

En la posterior versión generalizada de este modelo (GARCH), se asume que la

31. Autoregressive Conditional Heteroscedasticity (modelos Autorregresivos con Heteroscedasticidad Condicional). 
varianza de los rendimientos financieros sigue un proceso predecible. Este tipo de modelos tiene un carácter parsimonioso, con pocos parámetros, el cual se ajusta bien a los datos financieros. La característica básica de poseer varianzas condicionales que dependen de la última información incorporada es lo que permite utilizarlos con amplio éxito en el análisis de series de tiempo financieras, las cuales muestran sistemáticamente agrupaciones de volatilidad. En términos muy generales, lo anterior demuestra la no-normalidad, debido a la existencia de varianzas que cambian en el tiempo, en lugar de una varianza única y finita.

Además, los premios Nobel de Economía de 2005, Aumann y Schelling, y de 2007, Hurwicz, Maskin y Myerson, retoman el enfoque no-lineal de la Teoría de Juegos y lo aplican a la resolución de conflictos sociales, en el primer caso, y al desarrollo de la Mechanism Desing Theory (junto con los desarrollos de la Teoría de la Información), en el segundo caso.

Los anteriores desarrollos demuestran que, en el presente, la ciencia económica está dando un giro importante hacia la economía financiera y hacia la interdisciplinariedad (véase por ejemplo la obra de Peters, 1996, en la cual se desarrolla una aplicación de la Teoría del Caos a los mercados financieros), ya que seis de los últimos once premios Nobel de Economía entregados hasta 2005, han recibido dicho premio por aportes directos al campo financiero (véase nuevamente la Tabla 5). Esto también prueba que los mercados financieros son un campo importante de la economía el cual está siendo fuertemente investigado y estudiado en la actualidad.

Como comentario final se puede plantear que, la investigación científica se ha vuelto cada vez más interdisciplinaria efectivamente; por lo tanto, para poder explicar mejor muchos de los comportamientos actuales de la economía en general y de la Economía Financiera en particular, se han venido desarrollando teorías e hipótesis provenientes de la física cuántica, de la biología, de la psicología y de la neurociencia como lo son la Teoría del Caos, la Complejidad, la Hipótesis del Mercado Fractal, los algoritmos genéticos, la TCF y las redes neuronales artificiales, entre otros, que tratan de explicar los mercados financieros y la economía real desde una visión de no-linealidad, no-equilibrio e ineficiencia.

\section{PROPUESTA CURRICULAR EN EL ÁREA DE MERCADOS FINANCIEROS PARA COLOMBIA}

Actualmente, un estudiante de pregrado o postgrado de una escuela de negocios de una universidad americana acreditada tiene varias ventajas físicas importantes dentro del campus universitario; entre las más relevantes están: el acceso electrónico y físico a las publicaciones más recientes de las revistas académicas líderes en el campo financiero, libre acceso a los libros de texto de más reciente publicación, laboratorios financieros y acceso totalmente libre a una amplia red de computadoras con software y conexión de banda ancha alrededor del campus (esto incluye computadoras en pasillos, cafeterías, salones, además de las salas de cómputo y los equipos regulares de la biblioteca) ${ }^{32}$. Inicialmente se podría pensar

32. El Autor tuvo la oportunidad de visitar en el segundo semestre de 2004 seis escuelas de negocios (cuatro privadas y dos estatales) de algunas universidades estadounidenses acreditadas dentro de las que están Chicago University, University of Minnesota at Minneapolis, Columbia University, University of Nebraska Lincoln, Northwestern University y New York University. De acuerdo con esta visita, conversaciones sostenidas con algunos directivos, académicos y asistencia a algunas clases de nivel de maestría y doctorado, el 
que este contexto ayuda, en gran parte, al acercamiento a la frontera de investigación por parte de estudiantes y docentes; a esto se suman las condiciones socioeconómicas favorables del estudiante estadounidense y la fuerte cultura de investigación de muchas universidades americanas ${ }^{33}$. El anterior contexto debería ser un modelo a tener en cuenta por las universidades en Colombia.

De acuerdo con Borokovich y otros (2000) el área académica de mercados financieros es el área más importante para escribir investigaciones científicas académicas. De igual manera, siguiendo a Cooley y otros (1986), el tema de inversiones es el más importante para estudiar en los seminarios de docto- rado en finanzas. Lo anterior establece la necesidad de mejorar el currículo tradicional de finanzas que se dicta normalmente en algunas universidades en Colombia ${ }^{34}$, y ofrecer nuevos cursos en el área de Mercados Financieros, que, sin importar que sean para un nivel de pregrado o postgrado, tengan en cuenta el contexto de la evolución de la ciencia económica actual. Los cursos propuestos serían los siguientes ${ }^{35}$ : Mercado de Capitales Internacional, Finanzas Personales, Análisis Financiero Cuantitativo, Análisis de Riesgo de Mercado, Teoría Moderna del Portafolio, Política Económica y Financiera, Análisis Técnico Bursátil ${ }^{36}$, Teoría del Comportamiento Financiero, Mercados

Autor pudo obtener algunas conclusiones importantes sobre la vida en el campus universitario, el proceso de investigación y el acceso a la información que considera importante que puedan ser aplicadas, en la medida en que las posibilidades lo permitan, si no en todas, al menos en algunas de las escuelas de negocios en Colombia.

33. En los últimos años cada vez se le está exigiendo un mayor nivel y preparación académica a los estudiantes que aplican a los estudios de Maestría y Doctorado en Finanzas en las universidades estadounidenses; estos deben tener unas sólidas bases principalmente en matemática, estadística, econometría y macroeconomía. Los estudiantes de doctorado y los profesores deben publicar al menos dos artículos por año en revistas acreditadas. Por otra parte, en los últimos años, aproximadamente el $90 \%$ de los estudiantes que ingresan a programas de $\mathrm{Ph}$.D. en Finanzas en las universidades estadounidenses son extranjeros y del total de estudiantes admitidos aproximadamente el $70 \%$ estudia con beca otorgada por la universidad que lo admite (esto para el caso de las universidades estatales, en el caso de las privadas las becas son escasas). El criterio de admisión más relevante a un programa de Ph.D. en Finanzas es el puntaje obtenido, por parte del aspirante, en el GMAT (General Management Admission Test).

34. Esta afirmación se hace de acuerdo con la revisión de los programas académicos de Especialización en Finanzas de la Universidad Javeriana, Universidad Autónoma, Universidad del Valle, Universidad de los Andes, Universidad Externado, del programa académico del Diplomado Bursátil que ofrece la Bolsa de Valores de Colombia y de los programas académicos de Maestría en Finanzas de la Universidad EAFIT y la Universidad ICESI (en convenio con IIT). En la actualidad instituciones académicas como la Universidad del Valle y la Universidad Libre, entre otras, están tratando de crear sus programas de Maestría en Finanzas sin tener en consideración plena las recomendaciones que se plantean en este documento.

35. Esta propuesta de cursos está basada en la revisión por parte del Autor de los programas académicos de Doctorado y Maestría en Finanzas que se dictan actualmente en la University of Nebraska Lincoln, University of Minnesota at Minneapolis, Chicago University, el programa académico de algunos de los cursos que ofrece la Chicago Mercantile Exchange (CME) y el New York Institute of Finance (NYIF) y el resultado del proceso de investigación desarrollado por parte del Autor en esta área; para complementar la parte académica con la parte práctica (comercial) en esta área de las finanzas, el Autor se basa en su experiencia profesional obtenida en las compañías financieras UBS Warburg (Nueva York), Global Financial Services (Cali) y Massachusetts Financial Services (Boston) en las que tuvo anteriormente la oportunidad de trabajar como empleado directo y como independiente.

36. Aunque el Análisis Técnico Bursátil no ha sido tradicionalmente un área estrictamente académica sino más bien comercial, en años recientes Wisconsin University, MIT, entre otras instituciones académicas universitarias, están comenzando a aplicar programas similares a los que se ofrece en el NYIF o en la CME, utilizando los estándares desarrollados por la MTA (Market Technicians Association, http://www.mta.org). 
Derivados, Teoría de Caos y Mercado de Capitales, Asimetrías de Mercado, Historia Bursátil y Financiera, Redes Neuronales Artificiales Aplicadas a Bolsa, Globalización Financiera y Crisis Bursátiles. Los anteriores cursos tendrían su soporte en revistas académicas $^{37}$ (siguiendo los criterios de selección de artículos de acuerdo con la fecha de publicación presentado por Corrado y Ferris, 1997, (véase la página 8), libros de texto actualiza$\operatorname{dos}^{38}$ y desarrollos y aplicaciones prácticas en un laboratorio de análisis bursátil ${ }^{39}$. Lo anterior sugiere el estudio de varios temas que actualmente no están siendo estudiados en los programas académicos en el área de Finanzas y Economía en Colombia, y si se estudian algunos, se estudian sin tener en cuenta un enfoque interdisciplinario y científico. Entre algunos de estos temas, aplicados especialmente a la predicción de tendencias de los mercados financieros y bursátiles, están los siguientes: futuros de índices, árboles binomiales para valuación de opciones, exponentes de Lyapunov, análisis espectral, dimensión fractal, redes neuronales artificiales (backpropagation), osciladores

37. Actualmente varias universidades en Colombia tienen una suscripción completa a la base de datos electrónica http://www.jstor.org, adicional a esto, las bibliotecas universitarias deberían inscribirse a los tres o cuatro revistas más importantes en el campo financiero (JOF, JFE, JFQA y RFS) para los últimos cinco años, para así obtener la información de frontera sin demora (refiérase al "moving wall” de la base http:// www.jstor.org en la página 4). Sin embargo, es importante tener la suscripción completa a otras bases de datos electrónicas que permitan acceder a las revistas que no tiene http://www.jstor.org, estas podrían ser: http://www.elsevier.org, http://www.ebsco.org y http://www.sciencedirect.com; la intención es que, a la larga se tenga pleno acceso en línea a la totalidad de las publicaciones, en este caso, de las revistas más importantes en el campo financiero. Otro aspecto importante para los investigadores es que se puede tener acceso gratuito a los artículos publicados por parte de los profesores de las universidades estadounidenses a través de la página web de la correspondiente universidad, en el link del profesor, haciendo una petición formal por e-mail al profesor respectivo y justificando adecuadamente la necesidad de tener un determinado artículo. Si el profesor acepta dicha petición entonces envía dicho artículo por e-mail en formato pdf. Es muy importante tener en cuenta, para las diferentes áreas de investigación en el campo financiero, la bibliografía más relevante utilizada en los artículos escritos por los premios Nobel de Economía respectivos y en los artículos claves en el desarrollo de la Economía Financiera puesto que esta es la bibliografía consultada precisamente por los premios Nobel de Economía y los investigadores académicos y comerciales más importantes (ellos suelen citar las investigaciones publicadas por los premios Nobel de Economía y en algunos casos los premios Nobel de otras ciencias, y también artículos de autores muy importantes en cada campo de las finanzas respectivamente); además, la gran mayoría de los premios Nobel de Economía tuvieron, en su época de estudiante, a otros premios Nobel de Economía como profesores y, posteriormente, como colegas en las diversas investigaciones académicas realizadas a lo largo de su vida profesional y académica (para mayor información se puede consultar las biografías de los premios Nobel de Economía en http://www.nobel.se).

38. Para lograr esto las bibliotecas universitarias deben conseguir la lista de textos sugerida la cual debe contener libros de texto académicos y comerciales en cada una de las áreas respectivas. Esta se puede pedir a través de una librería en línea como http://www.amazon.com (para abaratar costos, a través de esta librería virtual se pueden pedir textos de segunda mano en muy buen estado) o en http://www.sciencedirect.com (realizando una suscripción electrónica para algunos de los libros de texto solicitados).

39. El laboratorio de análisis bursátil debe contener como mínimo: 15 computadores con licencias de un software financiero (Bloomberg Professional), un software para predicción con redes neuronales (Neuroshell), un software para programación y predicción bursátil (TradeStation), un software para realizar operaciones intradiarias (Neuroshell day-trading Professional), pantalla del Nasdaq (Nasdaq Level II), acceso a cotizaciones de buena fuente y en tiempo real (Quote.com, Economática.com), una pantalla digital grande, red de banda ancha con fibra óptica o satélite y acceso a noticias financieras 24 horas al día (Bloomberg TV). Además, en dicho laboratorio se debe aprender a negociar, a manejar clientes, a realizar consultoría y otras actividades que involucren la experiencia real del medio financiero, con salidas de campo, entrevistas y estudios de casos reales. 
técnicos normalizados, indicadores técnicos bursátiles, sistemas operacionales automáticos discrecionales, análisis intermercados, sesgos heurísticos, dependencia del contexto, psicología bursátil, índices bursátiles internacionales, burbujas y quiebras bursátiles, modelos ARCH, modelos ARIMA, series de Furier, simulaciones Monte Carlo estructurado, método de máxima entropía, algoritmos genéticos, lógica difusa, aprendizaje de máquina.

En el corto plazo, lo anterior implicaría una adecuada auto capacitación ${ }^{40}$ por parte de los docentes en formación, en este caso pertenecientes a un departamentos de finanzas, principalmente en el dominio del inglés hablado y escrito, en una fuerte utilización de las revistas académicas, en el manejo de medios electrónicos y tecnologías multimedia y en desarrollar investigación interdisciplinaria teórica y práctica en cada uno de sus campos respectivos en la frontera de la ciencia.

Pensando a más largo plazo, y con la intención de desarrollar un programa académico serio en el área de finanzas, que incluya una maestría en Finanzas inicialmente y posteriormente un doctorado en Finanzas, soportado por investigación y publicaciones fuertes, sería muy apropiado que los departamentos de Finanzas de las universidades colombianas interesadas enviaran con una beca parcial o total, al menos, a tres o cuatro de sus profesores en formación (que tengan el perfil y el compromiso) a realizar estudios de Ph.D. en Finanzas en alguna de las universidades estadounidenses de más alto prestigio (véase nuevamente la Tabla 1). Al terminar sus estudios de doctorado esos tres o cuatro profesores deben llegar a producir intelectualmente, de una manera similar a como se produce en las universidades estadounidenses, desarrollando proyectos de investigación, cursos nuevos, una mayor exigencia a los estudiantes, etcétera; y la propia universidad se debe encargar de mantener, ante todo, un ambiente propicio y recursos económicos para la investigación y el desarrollo académico, de tal manera que esos profesores puedan dar todo su potencial y retroalimentar a la comunidad académica local.

40. Para lograr esto, la universidad debe crear incentivos y estrategias serias de índole monetaria o académica con las cuales el docente realmente sienta que la auto capacitación vale la pena para él y para la comunidad. Este proceso debe ser supervisado adecuadamente y se debe constatar con resultados que el docente realmente está haciendo el esfuerzo de auto capacitarse. Como aporte a la docencia el profesor debe diseñar clases buenas y actualizadas, debe dominar la totalidad de los temas durante la clase, debe conseguir bibliografía de punta para la clase (preferiblemente originales y no traducciones), debe mejorar el material utilizado para docencia haciendo uso de tecnologías multimedia, debe crear cursos nuevos o mejorar los existentes y debe mantener un contacto con el medio comercial respectivo de la comunidad y retroalimentarlo en la clase para que lo que enseñe no quede solamente en teoría. Como aporte a la investigación, el profesor debe desarrollar investigación interdisciplinaria actualizada, estudiando cuál es el aporte de las demás ciencias a su respectivo campo de investigación, debe tener un buen dominio del inglés en todas sus formas $y$, si es posible, dominar varios idiomas, debe tener una experiencia profesional no académica correlacionada con el tema que investiga y enseña y finalmente debe, sobre todo, publicar sus investigaciones (preferiblemente libros de texto científicos y artículos científicos).

Lo anterior sugiere que para tener una adecuada retroalimentación de la auto capacitación del docente, el estudiante también debe adquirir algunos deberes sencillos: debe ir bien preparado a las clases (hacer una buena lectura del material obligatorio, tener preguntas concretas y estudiadas), debe asumir responsablemente el aprendizaje (realizar talleres, investigaciones, consultas; si falta a clase se debe nivelar rápidamente, debe mejorar su nivel de inglés en todas sus formas) y debe consultar nuevo material bibliográfico por su cuenta (textos, revistas, videos, noticias, sitios web). 


\section{CONCLUSIONES}

El estudio desarrollado por Corrado y Ferris (1997) tiene siete años y medio de haber sido publicado. Por lo tanto se asume que los resultados de dicho estudio no han cambiado de una forma significativa $y$, por consiguiente, aún son bastante útiles para aplicarlos al mejoramiento de la calidad de los programas en el campo financiero en Colombia.

El presente artículo es un breve catálogo de los mejores y más utilizados artículos de investigación académica en el área financiera, con la finalidad de ayudar a desarrollar una revisión bibliográfica densa, desde los orígenes hasta los más recientes desarrollos teórico-prácticos, en las diferentes áreas de las finanzas, a partir de dichas publicaciones. Este artículo está enfocado principalmente al campo financiero específicamente, al aporte de los premios Nobel de Economía al desarrollo de la Economía Financiera, especialmente en el área de Mercados Financieros.

De manera general se concluye que en el presente las revistas más importantes en el campo financiero son: JOF, JFE, RFE, JFQA y JOB respectivamente. Todos estos, con excepción del JFE, se consiguen a través de la base de datos electrónica http://www. jstor.org. En segundo lugar, en estas revistas, publican muchos de los docentes-investigadores de las universidades más importantes del mundo, que de acuerdo con el criterio de cantidad de publicaciones y premios Nobel de Economía recibidos, son respectivamente: Chicago University, University of California at Berkeley, Harvard University y Princeton University. En tercer lugar, la ciencia económica ha dado un giro importante hacia la Economía Financiera y hacia la no-linealidad y así lo demuestran las investigaciones de algunos de los premios Nobel de Economía más recientes, entre los que están Robert Aumann, Thomas Schelling, Robert Engle, Clive Granger, Daniel Kahneman, Vernon Smith, George Akerlof y Joseph Stiglitz. En cuarto lugar, la Economía Financiera ha dado un giro importante de la TMP a la TCF mostrando que los mercados son ineficientes e imperfectos. Finalmente, en el campo financiero, el área de Mercados Financieros es el área más importante para ser estudiada; propone cursos nuevos, desarrolla investigación y publica.

Una fuente importante en la actualidad, del alto prestigio académico en el campo financiero de universidades como Chicago, Harvard, Stanford, MIT, Yale, Berkeley, Columbia, Princeton es su alta productividad en investigación académica y científica registrada en un gran número de publicaciones en revistas académicas, tal como lo establece particularmente el estudio llevado a cabo por Cooley y otros (1986) y Klemkosky y Tuttle (1977). De acuerdo con estas dos investigaciones y a la desarrollada por Borokhovich y otros (1994), la Universidad de Chicago es, en la actualidad, la institución que más investigación y producción intelectual tiene en el campo financiero, muy por encima de las demás universidades estadounidenses y del mundo. De otra parte, y este es un aspecto sumamente importante, dichas universidades han obtenido la mayoría de los premios Nobel de Economía que se han otorgado hasta el presente (véase nuevamente la Tabla 4).

Al hacer una revisión de la bibliografía de varios artículos seleccionados al azar y 
escritos recientemente en el campo financiero (últimos tres años), se puede apreciar, en general, que un alto porcentaje de dicha bibliografía pertenece a publicaciones recientes, lo cual sugiere dos cosas: la necesidad de disponer de una base de datos plenamente actualizada y la necesidad de haber desarrollado un recorrido bibliográfico completo en cada campo de investigación de manera que se pueda establecer que, si no se menciona alguna investigación antigua relevante, que no suceda esto por desconocimiento de la misma. La complejidad de muchos artículos importantes (dentro de los que se encuentran los reseñados en el presente artículo) hace que con el paso del tiempo la investigación se reedite en un libro de una manera totalmente digerible a un público no especializado; sin embargo, en el ámbito académico es de suma importancia remitirse a los textos originales para, a partir de estos, abordar los nuevos desarrollos. Otro aspecto relevante respecto de la importancia del uso de las revistas consiste en que la gran mayoría de las publicaciones de texto serias de la actualidad tienen un gran componente de revistas en sus referencias bibliográficas y no solamente otros libros de texto.

Las investigaciones desarrolladas por los premios Nobel de Economía, marcan un nuevo aporte en la frontera del conocimiento que se sigue mejorando año tras año. Por consiguiente, poder acceder a los documentos originales de dichas investigaciones, así como a las referencias bibliográficas de dichas investigaciones y a las posteriores publicaciones de dichos autores, incluidas algunas de las más recientes, es un gran aporte para irse acercando a la frontera actual del conocimiento y con mayor razón si dichas investigaciones y líneas de investigación están, en su mayoría, de forma gratuita en la red. Por tanto, se considera de gran importancia que todos los docentes en el área de finanzas comiencen a incorporar masivamente las revistas como material obligatorio de lectura y discusión de clase (tanto en pregrado como en postgrado), con la finalidad de mejorar paulatinamente los cursos que se dictan. Lo anterior también permite la posibilidad de elaboración de artículos científicos (a partir de dichas referencias) más próximos a la frontera de la ciencia.

Aunque este documento se ha basado principalmente en material académico y temático utilizado en los estudios de doctorado en Finanzas, es precisamente en este nivel donde surge y se mueve la frontera del conocimiento; por lo tanto, los demás niveles (maestría, especialización, diplomados, pregrado) se alimentan de lo que surge en los doctorados en Finanzas. De esta forma, no es inapropiado de ninguna manera desarrollar los demás niveles a partir de lo que se está haciendo en el nivel de doctorado en Finanzas.

\section{BIBLIOGRAFíA}

- Akerlof George A. (1970): “The market of 'lemons': qualitative uncertainty and the market mechanism", Quarterly Journal of Economics, Vol. 84, No. 3, August, pp. 488-500.
- Bertin William J. y Zivney Terry L. (1992): "Publish or perish: what the competition is really doing", The Journal of Finance, Vol. 47, No. 1, March, pp. 295-329. 
- Borokhovich Kenneth A.; Bricker Robert J. y Simkins Betty J. (2000): "An Analysis of Finance Journal Impact Factors", The Journal of Finance, Vol. 55, No. 3, June, pp. 14571469.

- Borokhovich Kenneth A.; Bricker Robert J. Simkins Betty J. (1994): "Journal Communication and Influence in Financial Research", The Journal of Finance, Vol. 49, No. 2, June, pp. 713-725.

- Cooley Philip L.; Heck J. Louis Hubbard Carl M. (1986): "Contributing Authors and Institutions to the Journal of Finance: 1946-1985", The Journal of Finance, Vol. 41, No. 5, December, pp. 1129-1140.

- Corrado Charles, J. y Ferris Stephen P. (1997): "Journal Influence on the Design of Finance Doctoral Education", The Journal of Finance, Vol. 52, No. 5, December, pp. 20912102.

- Díaz Fernández Javier (2000). Dinámica caótica en economía, McGraw-Hill, Madrid.

- Engle Robert F. (1982): "Autoregressive conditional heteroscedasticity with estimates of the variance of United Kingdom inflation", Econométrica, Vol. 50, No. 4, pp. 987-1008.

- Frankfurter, George M.; McGoun Elton y Allen Douglas (2004): "The prescriptive turn in behavioral finance", The Journal of SocioEconomics, No. 33, pp. 449-468.

- Guthrie, Kevin M. (2001): “Archiving in the digital age: there's a will, but is there a way?" Educase Review, Vol. 36, No. 6, November/December, pp. 5665.
- Kahneman, Daniel; Tversky Amos (1979): "Prospect Theory: An Analysis of Decision under Risk", Econométrica, Vol. 47, No. 2, March, pp. 263-292.

- Klemkosky, Robert C.; Tuttle Donald L. (1977): "A Ranking of Doctoral Programs by Financial Research Contributions of Graduates", The Journal of Financial and Quantitative Analysis, Vol. 12, No. 3, September, pp. 491-497.

- Kurtzman, Joel (1993): The death of money. How the electronic economy has destabilized the world's markets and created financial chaos, Back Bay Books, USA, pp. 256.

- Lindbeck Assar (1985) "The Prize in Economic Science in Memory of Alfred Nobel”, Journal of Economic Literature, Vol. 23, No. 1, March, pp. 37-56.

- Monroy D’Croz, Julián D. (2005): Complejidad y mercados bursátiles, Tesis de Grado presentada a la Maestría en Ciencias de la Organización, Facultad de Ciencias de la Administración, Universidad del Valle, Cali, pp. 148.

- Peters, Edgar E. (1996): Chaos and Order in the Capital Markets: A New View of Cycles, Prices, and Market Volatility, $2^{\text {nd }}$ Edition, New York: John Wiley.

- Shefrin, Hersh (2000): Beyond greed and fear, understanding behavioral finance and the psychology of investing, Harvard Business School Press, pp. 424.

- Stiglitz, Joseph E. y Grossman Sanford J. (1980). "On the Impossibility of Informationally Efficient Markets, The American Economic Review", Vol. 70, No. 3, June, pp. 393-408. 